\title{
Stress-driven integration strategies and m-AGC tangent operator for Perzyna viscoplasticity and viscoplastic relaxation: application to geomechanical interfaces
}

\author{
I. Aliguer ${ }^{1, *, \dagger}$, I. Carol ${ }^{1}$ and S. Sture ${ }^{2}$ \\ ${ }^{1}$ Geotechnical Engineering and Geo-Sciences Department (DETCG) ETSECCPB (School of Civil Engineering), UPC \\ (Universitat Politécnica de Catalunya), 08034 Barcelona, Spain \\ ${ }^{2}$ Department of Civil, Environmental, and Architectural Engineering, University of Colorado at Boulder Boulder, CO \\ 80309, U.S.A.
}

\begin{abstract}
SUMMARY
The paper proposes a stress-driven integration strategy for Perzyna-type viscoplastic constitutive models, which leads also to a convenient algorithm for viscoplastic relaxation schemes. A generalized trapezoidal rule for the strain increment, combined with a linearized form of the yield function and flow rules, leads to a plasticity-like compliance operator that can be explicitly inverted to give an algorithmic tangent stiffness tensor also denoted as the m-AGC tangent operator. This operator is combined with the stress-prescribed integration scheme, to obtain a natural error indicator that can be used as a convergence criterion of the intrastep iterations (in physical viscoplasticity), or to a variable time-step size in viscoplastic relaxation schemes based on a single linear calculation per time step. The proposed schemes have been implemented for an existing zero-thickness interface constitutive model. Some numerical application examples are presented to illustrate the advantages of the new schemes proposed. Copyright (C) 2016 John Wiley \& Sons, Ltd.
\end{abstract}

Received 7 December 2015; Revised 15 September 2016; Accepted 11 October 2016

KEY WORDS: viscoplasticity; viscoplastic relaxation; finite element method; interface elements

\section{INTRODUCTION}

Viscoplasticity has been widely used for engineering materials with (physical) time-dependent behavior over a threshold stress level $[1,2]$ or in the context of viscoplastic relaxation (VPR) strategies to obtain the stationary solution of an inviscid problem via a fictitious (non-physical) pseudo-time [3-5]. In either case, the rate-type infinitesimal viscoplastic formulation requires a time integration strategy to (a) discretize time in increments and (b) evaluate a linearized relation between stress and strain increments for each time step and, possibly, some residual force calculation and iterative strategy. Typically, the algorithms are based on the initial stress scheme used in FEs, in which the strain increments are prescribed to the constitutive equations. A variety of such algorithms has been proposed since the original constant stiffness and constant stress procedures [6-8] to more recent and sophisticated contributions [9-11] . Other research has been developed incorporating a consistency condition to the traditional viscoplastic strain rate formulation [12-15].

In contrast, stress-driven schemes are not that common. After the original constant-stress implementation of Zienkiewicz and Cormeau [1] that may be considered the most elementary form of stress-driven viscoplastic schemes, to the knowledge of the authors, the only previous work of this type is [16]. The implementation of stress-prescribed schemes are conceptually much simpler and

*Correspondence to: I. Aliguer, Geotechnical Engineering and Geo-Sciences Department (DETCG) ETSECCPB (School of Civil Engineering) UPC (Universitat Politécnica de Catalunya), 08034 Barcelona, Spain.

${ }^{\dagger}$ E-mail: ignasi.aliguer@upc.edu 
numerically advantageous (explicit integration of the constitutive equations and simple coding). However, in the original form, their stability was related to the size of the time step, and for small time steps, large number of iterations may be required.

The type of viscoplastic formulation also conditions significantly the integration strategy. For Duvaut-Lions formulations, quasi-linear exponential algorithms and consistent viscoplastic tangent operators lead to a good compromise between complexity and computational cost [17-19]. However, for Perzyna viscoplasticty, there seems to be no equivalent approach, in spite of the existing literature, on the order of the integration strategies [13, 20], time-step discretization [21, 22] and tangent operators $[23,24]$.

The main objective of this paper is to describe and demonstrate a stress-prescribed integration algorithm for Perzyna viscoplasticity in an FE framework. Among the main advantages of the proposed integration algorithm, which is applicable to both physical viscoplasticity and to VPR, is that it exhibits a closed-form consistent tangent stiffness operator, leading to a better compromise between accuracy of the solution and number of iterations.

In the field of rock mechanics, time dependence was incorporated in early models for the overall homogenized behavior of rock masses and rock interfaces, often associated to the creep phenomena or salt formations [25-29]. The use of viscoplasticity for general rock masses became common after the proposal and development of the Multilaminate model $[26,30]$. The numerical examples presented in this paper represent academic problems of rock samples with discontinuities. The rock matrix, represented by continuum elements, is considered linear-elastic, while the discontinuities are represented in a discrete manner using zero-thickness interface elements equipped with viscoplastic behavior. The viscoplastic algorithms developed in the paper are applied to those interface elements.

After this introduction, the content of the paper is structured as follows: Section 2 describes the basic concepts and expressions of Perzyna viscoplasticity. Section 3 develops the main assumptions and equations of the proposed stress-prescribed algorithm, leading to the tangential algorithmic compliance and stiffness operators for a fixed time increment. Section 4 describes the application of the algorithm to VPR schemes, in which time is not physical time, but the fictitious time used in this kind of procedures. In Section 5, the stress-prescribed integration algorithm is specified for the type of constitutive laws used for zero-thickness interface elements and in particular for a hyperbolic loading function in terms of stress tractions and relative displacements. Several examples of increasing complexity are presented, which show the favorable performance of the algorithm presented. Finally, Section 6 summarizes the main achievements and conclusions of the paper. Some specific aspects of the formulation presented are included in Appendices A and B at the end of the paper.

\section{PERZYNA VISCOPLASTIC CONSTITUTIVE MODEL}

Within the classical framework of small strain Perzyna viscoplasticity, total strain $\epsilon_{i j}$ can be additively split into the elastic $\epsilon_{i j}^{e l}$ and the viscoplastic $\epsilon_{i j}^{v p}$ :

$$
\epsilon_{i j}=\epsilon_{i j}^{e l}+\epsilon_{i j}^{v p}
$$

The elastic strain tensor is related to stress by considering isotropic linear elasticity:

$$
\epsilon_{i j}^{e l}=D_{0}^{-1} i j k l \sigma_{k l}
$$

where $\boldsymbol{D}_{0}$ is an elasticity stiffness matrix, symmetric, and positive definite. $\boldsymbol{D}_{0}^{-1}$ denotes its inverse, that is, the elasticity compliance matrix, which will be referred to as $\boldsymbol{C}_{\mathbf{0}}$, and $\sigma_{i j}$ is the stress tensor.

The loading function $F(\sigma)$ is defined to distinguish between elastic states $(F \leq 0)$ and viscoplastic states $(F>0)$. In the latter case, the classical Perzyna viscoplastic strain rate is considered: 


$$
\dot{\epsilon}_{i j}^{v p}=\frac{1}{\eta}\left\langle\psi\left(\frac{F(\sigma)}{F_{0}}\right)\right\rangle m_{i j}
$$

where the parameter $\eta$ is the viscosity of the material and $F_{0}$ is a reference value of the yield function, $Q$ the viscoplastic potential typical of non-associated formulations and the flow rule is $m_{i j}=\partial Q / \partial \sigma_{i j}$.

Finally, the accumulated viscoplastic strain $\epsilon_{i j}^{v p}$ in (1) can be obtained by integrating in time the viscoplastic strain rate:

$$
\epsilon_{i j}^{v p}=\int_{0}^{t} \epsilon_{i j}^{v p} d t=\int_{0}^{t} \frac{1}{\eta}\left\langle\left(\frac{F(\sigma)}{F_{0}}\right)\right\rangle m_{i j} d t
$$

\section{STRESS-DRIVEN INTEGRATION OF VISCOPLASTICITY AND m-AGC TANGENT OPERATOR}

A stress-driven integration scheme for Perzyna viscoplasticity is hereby developed. The main advantage is that, with the assumption of a known stress history, the integration of the constitutive relation is reduced to the numerical explicit evaluation of the integral expression (4). But the integration scheme for a finite element implementation also requires the linearized form of the constitutive tangential stiffness tensor. Each of those aspects, as well as a flow chart diagram illustrating how these ingredients fit into the general FE calculation, are given in the following sections.

\subsection{Explicit integration of Perzyna viscoplasticity with prescribed stress}

In the proposed integration scheme, it is assumed that the stress increment $\Delta \sigma$ that takes place during a time increment $\Delta t$ is known and that this increment is prescribed linearly in time, that is, at a fixed rate $\dot{\sigma}_{i j}=\Delta \sigma / \Delta t$. Note that if we assume that the stress evolution to be known, all the terms included in the integral expression (4) are also known, and therefore, the integral can be evaluated explicitly, using any quadrature rule with the general expression:

$$
\Delta \epsilon_{i j}^{v p}=\sum_{i=1}^{n q}\left[\frac{1}{\eta}\left\langle\left(\frac{F(\sigma)}{F_{0}}\right)\right\rangle m_{i j}\right]_{\left(\sigma=\sigma_{i}\right)} w_{i}
$$

with $\sigma_{i}=(\Delta \sigma / \Delta t)\left(t_{i}-t_{0}\right)$. In which the number of integration points $n q$, their position in the interval $t_{i}$, and weights $w_{i}$, will depend on the particular quadrature rule used. For each quadrature point $i=1, n q$ the integrand between brackets is evaluated for the corresponding stress as obtained from the linear variation law assumed within the increment.

As the quadrature rule, one may use a simple trapezoidal formula involving integrand values at both ends $\left(\int_{0-1} f(t) d t=\left(f_{0}+f_{1}\right) \Delta t / 2\right)$, a Simpson's rule using both ends and the middle point $\left(\int_{0-1} f(t) d t=\left(f_{0}+4 f_{1 / 2}+f_{1}\right) \Delta t / 6\right)$, or even more complex formulas using more intermediate points. However, in the calculations performed, there seems to be no need in general to go beyond Simpson's formula.

Note that in this scheme, the simple explicit evaluation of Equation (5) is all that has to be done to integrate the constitutive model. This is in contrast to the classical strain-driven algorithms of elastoplasticity that applied to this case become implicit and require subdivision of the increment into subincrements or iterate according to a Backward-Euler strategy. In the Appendix A, the integration of the viscoplastic constitutive relation for both stress-driven and strain-driven schemes is detailed. A simple example is also included, which illustrates the higher complexity in terms of number of operations needed in the strain-driven scheme.

\section{2. m-Assumed algorithmic generalized compliance tangent operator}

Additional to the integration of the constitutive model, the implementation into an FE code also requires the evaluation of a constitutive tangential stiffness tensor to be used in the first pseudoelastic linear calculation and in subsequent iterations. 
The integration of (4) can be discretized using a generalized trapezoidal rule for the interval $\Delta t_{n+1}=t_{n+1}-t_{n}$, with following expression:

$$
\Delta \boldsymbol{\epsilon}_{n+1}^{v p}=\mu_{n+1}\left((1-\theta) F\left(\sigma_{n}\right) \boldsymbol{m}\left(\sigma_{n}\right)+\theta F\left(\sigma_{n+1}\right) \boldsymbol{m}\left(\sigma_{n+1}\right)\right)
$$

where $\mu_{n+1}=\Delta t_{n+1} / \eta F_{0}$ groups the constant values of the increment. In this equation, the scalar factor $\theta$ may take a fixed value between 0 and 1. If $\theta=0$, the formula is equivalent to the forward Euler scheme, in which all variables are known at the beginning of the increment and therefore is no need of iterate within the time step. Early implementations of viscoplasticity used this approach with a fixed value of stiffness equal to the elastic stiffness. However, this scheme turns out very sensitive to the size of the time step, and it usually leads to slow convergence and a high number of iterations [6].

The other limit case is when $\theta=1$. In that case, the values of $F$ and $\boldsymbol{m}$ are those at the end of the interval, which corresponds to the traditional backward Euler scheme.

For any intermediate value $0<\theta<1$, the values of $F$ and $\boldsymbol{m}$ at both ends of the interval are needed with the weighting factors $\theta$ and $(1-\theta)$.

Note that, whenever $\theta>0$, the value of $\sigma_{n+1}$ is needed to evaluate (6). Therefore, in the context of a FE calculation, iterations are usually required within the time step (until the viscoplastic strain increments at each Gauss point are such that the overall stress redistribution leads to the prescribed stress increment).

Next step in the formulation is to linearize the yield function and the plastic potential around their values at the beginning of the time step:

$$
\begin{aligned}
& F\left(\sigma_{n+1}\right)=F\left(\sigma_{n}+\Delta \sigma_{n+1}\right)=F\left(\sigma_{n}\right)+\frac{\partial F}{\partial \sigma}\left(\sigma_{n}\right): \Delta \sigma_{n+1}+\ldots \\
& \boldsymbol{m}\left(\sigma_{n+1}\right)=\boldsymbol{m}\left(\sigma_{n}+\Delta \boldsymbol{\sigma}_{n+1}\right)=\boldsymbol{m}\left(\boldsymbol{\sigma}_{n}\right)+\frac{\partial \boldsymbol{m}}{\partial \boldsymbol{\sigma}}\left(\sigma_{n}\right): \Delta \boldsymbol{\sigma}_{n+1}+\ldots
\end{aligned}
$$

Considering now that $\partial F / \partial \sigma_{i j}=n_{i j}$ and replacing these expressions into (6), one obtains

$$
\begin{aligned}
\Delta \boldsymbol{\epsilon}_{n+1}^{v p}= & \mu_{n+1}\left(F_{n} \boldsymbol{m}_{n}+\theta \boldsymbol{m}_{n} \otimes \boldsymbol{n}_{n}: \Delta \boldsymbol{\sigma}_{n+1}+\theta F_{n} \frac{\partial \boldsymbol{m}}{\partial \boldsymbol{\sigma}_{n}}: \Delta \boldsymbol{\sigma}_{n+1}+\right. \\
& \left.+\theta \Delta \boldsymbol{\sigma}_{n+1}: \frac{\partial \boldsymbol{m}}{\partial \boldsymbol{\sigma}_{n}} \otimes \boldsymbol{n}_{n}: \Delta \boldsymbol{\sigma}_{n+1}\right)
\end{aligned}
$$

where for clarity $F\left(\sigma_{n}\right)$ has been replaced by $F_{n}$.

If the second order term is not taken into account and under the assumption that the variation of the plastic potential is small with respect to the variation of the stress within the interval, the simplified expression is obtained

$$
\Delta \boldsymbol{\epsilon}_{n+1}^{v p}=\mu_{n+1} F_{n} \boldsymbol{m}_{n}+\theta \mu_{n+1} \boldsymbol{m}_{n} \otimes \boldsymbol{n}_{n}: \Delta \boldsymbol{\sigma}_{n+1}
$$

By adding it to the elastic strain increment and grouping terms, one obtains the total strain increment expression:

$$
\Delta \epsilon_{n+1}=C_{n+1}^{\prime \prime}: \Delta \sigma_{n+1}+\Delta \epsilon_{n+1}^{\prime \prime}
$$

with,

$$
\begin{gathered}
\boldsymbol{C}_{n+1}^{\prime \prime}=\boldsymbol{C}_{0}+\theta \mu_{n+1} \boldsymbol{m}_{n} \otimes \boldsymbol{n}_{n} \\
\Delta \boldsymbol{\epsilon}_{n+1}^{\prime \prime}=\mu_{n+1} F_{n} \boldsymbol{m}_{n}
\end{gathered}
$$

in where the m-AGC tangent operator $\boldsymbol{C}_{n+1}^{\prime \prime}$ has been identified, as well as the initial strain term $\Delta \epsilon_{n+1}^{\prime \prime}$. Note that the latter corresponds to the viscoplastic strain that would take place during the time increment if the stress would have remained constant, that is, to the viscoplastic strain increment in the original constant-stress algorithms [1,2]. 
The tangent compliance $\boldsymbol{C}_{n+1}^{\prime \prime}$ may be rewritten in the alternative form

$$
\boldsymbol{C}_{n+1}^{\prime \prime}=\boldsymbol{C}_{0}+\frac{1}{H^{\prime \prime}} \boldsymbol{m}_{n} \otimes \boldsymbol{n}_{n}
$$

with $H^{\prime \prime}=1 / \theta \mu_{n+1}$, and in which, a tensorial structure identical to classical elastoplasticity [31] may be immediately recognized. Therefore, same as in that theory, the corresponding tangential stiffness can be derived explicitly via Shermann-Morrison formula [32] as

$$
\begin{gathered}
\Delta \boldsymbol{\sigma}_{n+1}=\boldsymbol{D}_{n+1}^{\prime \prime}:\left(\Delta \boldsymbol{\epsilon}_{n+1}-\Delta \boldsymbol{\epsilon}_{n+1}^{\prime \prime}\right) \\
\boldsymbol{D}_{n+1}^{\prime \prime}=\boldsymbol{D}_{0}-\frac{\boldsymbol{D}_{0}: \boldsymbol{m}_{n} \otimes \boldsymbol{n}_{n}: \boldsymbol{D}_{0}}{H^{\prime \prime}+\boldsymbol{n}_{n}: \boldsymbol{D}_{0}: \boldsymbol{m}_{n}}
\end{gathered}
$$

This explicit expression of the tangential stiffness tensor $\boldsymbol{D}_{n+1}^{\prime \prime}$ is one of the main advantages of the integration scheme proposed, because it eliminates the need of inverting the compliance tensor numerically at each Gauss point for each iteration, as is common for most existing integration schemes for viscoplastic models.

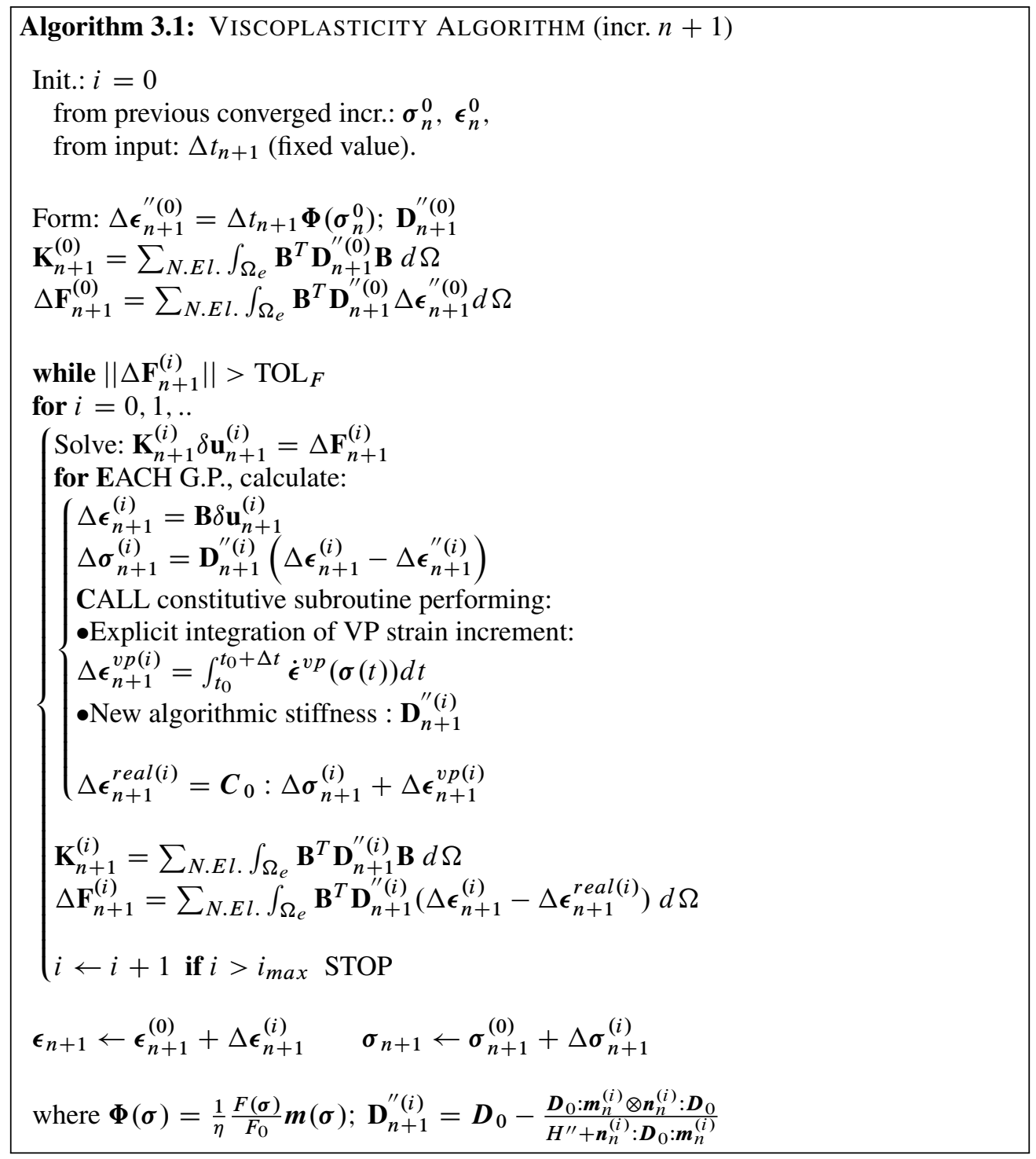


Indeed, if a tensorial structure different from (13) is assumed for the compliance tensor, the Shermann-Morrison formula is no longer applicable to obtain the explicit stiffness tensor as the inverse. For instance, by taking additional terms in the development of $\Delta \epsilon_{i j}^{v p}$, such as $\partial m_{i j} / \partial \sigma_{k l}$, one obtains more complex forms of the tangent compliance, which in principle might be more accurate. However, with the additional terms, the analogy of (15) to the elastoplastic stiffness is lost and so is the analytical closed-form for $\boldsymbol{D}$, which implies the need for numerical inversion of $\boldsymbol{C}$. It may of course be argued whether the extra computational cost may perhaps pay off in cases in which $Q$ varies rapidly within the increment, although in a large computation, this may affect to a limited number of Gauss points and may be compensated by some extra iterations, while there is no doubt that the savings in inverting the compliance matrix will benefit to all Gauss points in all iterations and therefore is a sure advantage, additional to the theoretical benefit itself of having an explicit stiffness tensor.

The numerical implementation of the proposed scheme within the framework of a finite element code is shown in Algorithm (3.1).

In the above algorithm, when $\theta=0$, the original explicit viscoplastic scheme by Zienckiewicz [1] is recovered, in which all the variables are evaluated at the beginning of the increment, and there is no need to iterate in the time increment to recalculate the initial stiffness (although already mentioned, this leads to the need of very small time increments).

On the other hand, for values of $\theta>0$, the accuracy of the viscoplastic strain calculated via (5) depends mainly on the accuracy of the linearization of the stress function (7). If the approximation is not accurate enough, the iterative algorithm will recalculate the viscoplastic strain at each iteration. Recalculating the stiffness at each iteration has a cost, but with the explicit evaluation of the tangent stiffness, this cost turns out a reasonable compromise; on one hand, it may be much lower compared with other schemes in which this calculation has to be done via compliance tensor first and then numerical inversion to obtain stiffness, and on the other hand, it will surely be much less expensive than the original constant stress/constant stiffness explicit scheme because of the much lower number of iterations.

\section{VISCOPLASTIC RELAXATION}

In the previous section, the viscoplastic model was considered as representing physical timedependent material behavior, that is, the time variable corresponded to the actual physical time. However, viscoplasticity has also been used in the literature as a numerical artifact to reach a final stationary state in which the stress state at all Gauss points lies on the plastic surface, that is, as a mere iterative procedure in the context of inviscid elastoplasticity, in which the fictitious time has the meaning of an always-increasing parameter leading to the stationary solution.

Algorithm 4.1: VPR (incr. $n+1)$

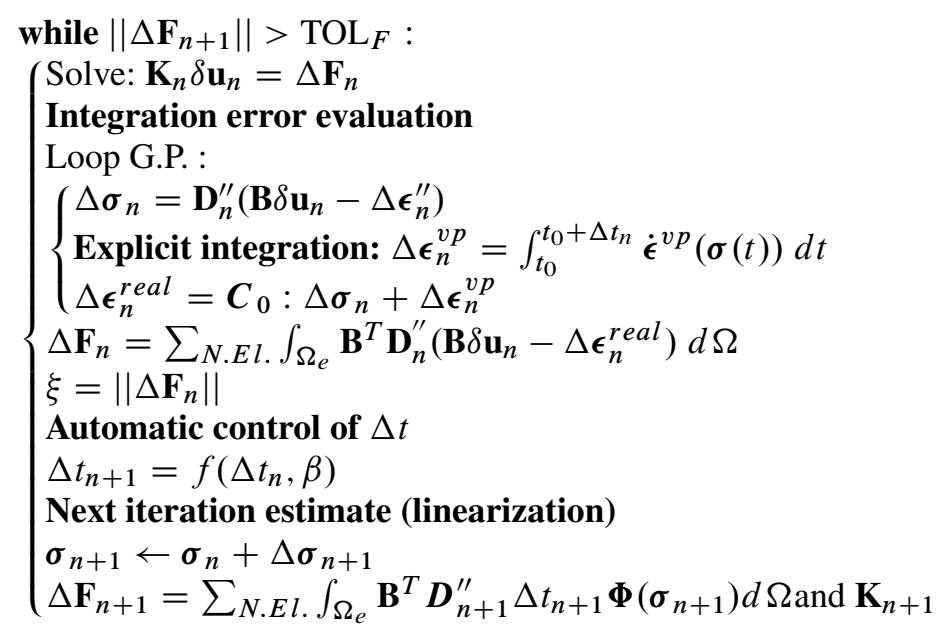


In the same philosophy, the integration scheme proposed in the previous section may be reformulated as a VPR scheme. In the algorithm for physical viscoplasticity, the time-step magnitude $\Delta t$ was considered as fixed a priori of the time-step calculation (and then if needed, iterations were performed on the corresponding stress and strain increments). Instead, for VPR, the magnitude of the time increment itself may be readjusted during iterations, from smaller time steps when the stress states are far from the plastic surface and strain rates are high to larger time steps when the stress points approach the surface and viscoplastic rates are lower.

The viscoplastic integration scheme proposed in the previous section may be easily readapted as a variable time-step VPR procedure, because it provides a natural way to evaluate the integration error. Indeed, the difference between the viscoplastic strain increment estimated via the linearized expression (15) and the viscoplastic strain resulting from the actual direct integration (4) that may be performed after the stress increment has been obtained at each Gauss point and provides an excellent measure of such error. Based on this, the variable time-step VPR scheme may be understood as if the time-step size would be readjusted automatically so that the linearized compliance (10) or stiffness (15) would be sufficiently approximate and no stress-strain iterations within the time increments would be ever needed.

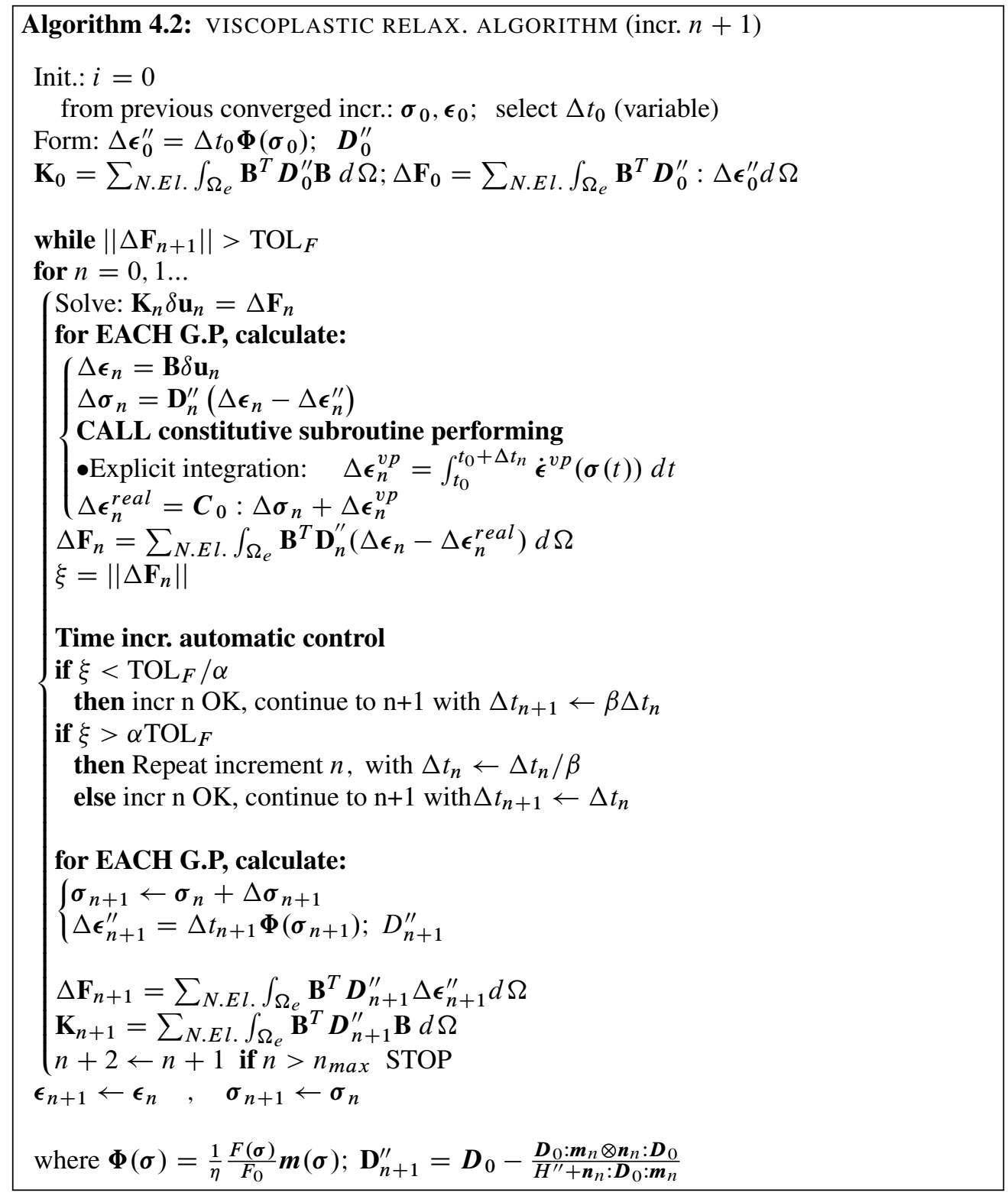


A detailed description of the VPR scheme is provided in Algorithm 4.2. Also, a brief summary that highlights the main features of the scheme within FE iterations is described as follows:

Note that, for each (pseudo) time step, the initial viscoplastic strain increment at each Gauss point coincides with the one for the traditional Zienckiewicz algorithm (using the value of the stress at the beginning of the increment $\sigma_{n+1}^{i}$ ), although if $\theta>0$ stiffness is the new explicit one (15) (if $\theta=0$ stiffness is also the elastic one and the algorithm coincides again with Zienckiewicz's with variable time step). Once the global (FE) system of equations is solved for $\delta \mathbf{u}_{n+1}^{i}$, the resulting increments of viscoplastic strain and stress at each Gauss point for the iteration $\left(\Delta \boldsymbol{\sigma}_{n+1}^{i}\right)$ may be recovered using Equations (9) and (15).

The automatic control on the time increment works as follows: once the stress increment has been obtained, the 'real' viscoplastic strain may be calculated using explicit integral (5), and both a priori and a posteriori viscoplastic strain increments may be compared. This comparison is performed at Gauss point level and then integrated and assembled into the accuracy index $\xi$. If this value remains between some minTOL and maxTOL tolerance values, the times step is accepted, and the following one is undertaken with the same size. If the index is lower than minTOL, the increment is accepted, but the next one is undertaken with a larger time step, and finally, if the index is greater than maxTOL, the increment is discarded and reattempted with a smaller time step.

The iterative procedure reaches convergence when the stress state is sufficiently close to the yield surface, and therefore, the residual stress is smaller than a tolerance value, which corresponds to stationary conditions.

\section{APPLICATION OF THE ALGORITHM TO INTERFACES}

In this section, the viscoplastic scheme described in previous paragraphs is implemented in the context of a constitutive model for zero-thickness interfaces based on hyperbolic loading surface. This model was proposed originally for the behavior of geotechnical interfaces [33], later modified for fracture energy-based opening and development of cracks in quasi-brittle materials (concrete, rock, etc) [34], and more recently extended to 3D and reformulated more efficiently [35]. For the viscoplastic implementation, the model is taken in its simplest format, that is, perfect plasticity and associated flow.

\subsection{Hyperbolic constitutive model}

The yield function $F$ is defined in terms of normal and shear stresses on the interface surface $\left(\sigma, \tau_{1}, \tau_{2}\right)$ and the strength parameters cohesion and friction angle $(c, \phi)$. The expression is

$$
F=-(a-\sigma) \tan \phi+\sqrt{\tau_{1}^{2}+\tau_{2}^{2}+a^{2} \tan ^{2} \phi}
$$

where $a$ is a new parameter related to $c, \phi$ as $a=c / \tan \phi$ (Figure 1(a)).

In this paper, the model is defined as elastic-perfectly plastic (fixed parameters $c, \tan \phi$ ). The flow rule is defined as non-associative, except in the case of pure tension. The specific definition of the flow rule is given as follows:

$$
\begin{aligned}
& \text { if } \quad \sigma \leq \sigma_{d i l} \longrightarrow \quad \boldsymbol{m}=\frac{1}{\sqrt{\tau_{1}^{2}+\tau_{2}^{2}}}\left(\begin{array}{c}
0 \\
\tau_{1} \\
\tau_{2}
\end{array}\right) \\
& \text { if } \quad \sigma>\sigma_{d i l} \longrightarrow \quad \boldsymbol{m}=\frac{1}{\sqrt{\left(\sigma-\sigma_{d i l}\right)^{2}+\tau_{1}^{2}+\tau_{2}^{2}}}\left(\begin{array}{c}
\left(\sigma-\sigma_{d i l}\right) \\
\tau_{1} \\
\tau_{2}
\end{array}\right)
\end{aligned}
$$

where $\sigma_{d i l}$ is a value of the compressive normal stress beyond which no dilatancy takes place. For compressive values of $\sigma<\sigma_{d i l}$, the flow rule used is radial with center at the point $\left(\sigma_{d i l}, 0\right)$, as depicted in Figure 1(b). 


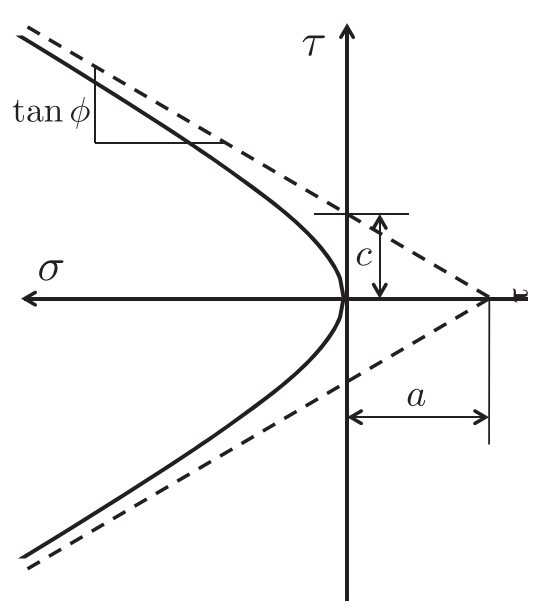

(a)

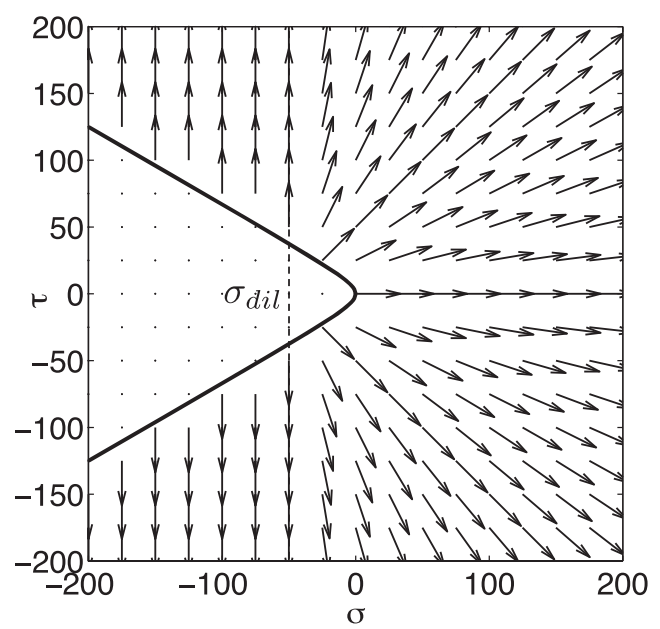

(b)

Figure 1. (a) Geometric definition of the yield surface in the $\sigma-\tau$ plane $\left(\tau=\sqrt{\tau_{1}^{2}+\tau_{2}^{2}}\right)$ and (b) representation of the non-associative flow rule $\left(\sigma_{d i l}=-50 \mathrm{kPa}\right)$.

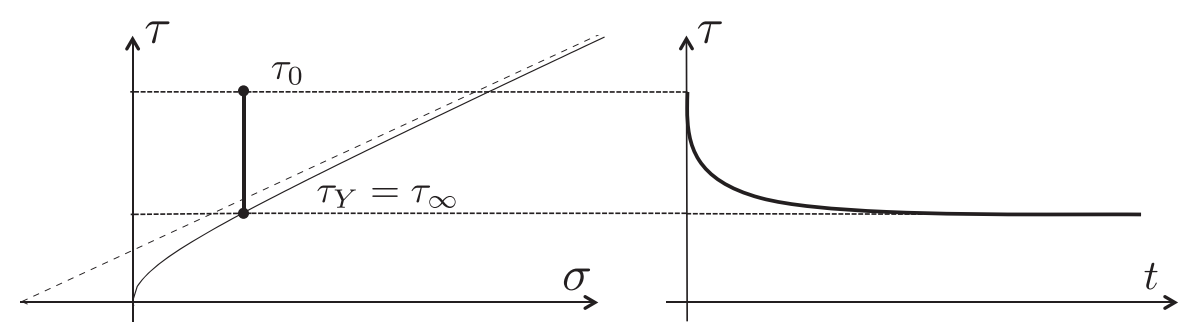

Figure 2. Shear-stress path in $\sigma-\tau$ space and qualitative shear stress evolution through time.

\subsection{Numerical examples}

Three simple examples are presented in this section in order to illustrate the features of the proposed formulation. The first example is a purely constitutive test and considers viscoplasticity with physical time. An analytical solution is available for comparison of all the integration strategies in this particular example. The second example is a semi-analytical pullout test computed in the context of a FEM calculation. The VPR scheme is now applied, and the solution obtained is compared with the one obtained with physical viscoplasticity. The third and final example consists on a geomechanical wedge failure mechanism with a prescribed displacement rate. In this case, the ability of the formulation to capture a viscoplastic behavior is discussed.

5.2.1. Relaxation test at the constitutive level. The first example of application consists of a relaxation test at the constitutive level. Normal stress is applied first, followed by prescribed shear relative displacement (at constant normal stress). Then, time is allowed to pass, and shear stress decreases to the limit value dictated by the inviscid yield surface (Figure 2).

The special case without cohesion $c=0$ in (16) is considered because for this particular case; it is possible to find the following closed-form expression for the decay of the shear stress with time $\tau=f(t)$ :

$$
\frac{\tau-\tau_{Y}}{\tau_{0}-\tau_{Y}}=\exp \left(\frac{-K_{T}}{\eta F_{0}} t\right)
$$




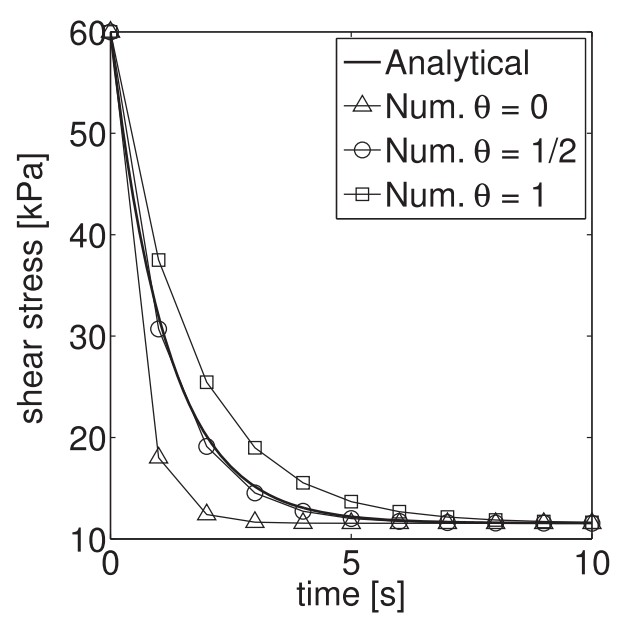

Figure 3. Analytical and numerical results for the evolution of the shear stress with time for $\Delta t=1 \mathrm{~s}$.

where $\tau_{0}$ is the value of shear stress at time $t_{0}, \tau_{Y}$ is the shear yield threshold value, and $K_{T}$ is the transversal stiffness modulus of the interface. Expression (18) indicates that, at the limit of infinite time, the value $\tau-\tau_{Y}$ tends to zero; that is, the shear stress tends to the shear stress yield limit value $\tau_{Y}$, and the elastoplastic solution is recovered.

The curves obtained with the aforementioned formula have been represented in Figure 3, together with the numerical results for the same case obtained numerically with $\Delta t=1 \mathrm{~s}$ and three different values of parameter $\theta=0,1$, and 0.5 .

In the numerical calculations, the load has been applied in two steps: (1) $\Delta u=-2 \cdot 10^{-6} \mathrm{~m}$ and $\Delta v=6 \cdot 10^{-6} \mathrm{~m}$ with $\Delta t=0 \mathrm{~s}$ to generate the initial stress state beyond the yield surface; and (2) $\Delta u=\Delta v=0$ and $\Delta t>0$. The second step has been subdivided in 10 increments to reach the final time of $t_{f}=10 \mathrm{~s}(\Delta t=1 \mathrm{~s})$. Material parameters used for this example are as follows: $K_{N}=K_{T}=10^{7} \mathrm{kN} / \mathrm{m} ; c=0.0 \mathrm{kN} / \mathrm{m}^{2} ; \tan \phi=0.577 ; \eta=10^{6} \mathrm{kPa} \mathrm{s}$.

The figure shows that the strategy with $\theta=1 / 2$ yields a much better approximation than $\theta=0$ or $\theta=1$. A measure of the overall error has been established as the difference of the area under the curves to the one corresponding to the analytical solution, as the total amount of viscoplastic strain will be approximately proportional to that area. The results obtained give an error lower than $1 \%$ for the strategy with $\theta=1 / 2$ and $14.1 \%$ and $14.0 \%$ for $\theta=0$ or $\theta=1$, respectively. As it would be expected, assuming constant stress for the increment equal to the initial value (forward scheme with $\theta=0$ ) leads to an over-prediction of the viscoplastic strain, and therefore, shear stress decreases faster. However, assuming $\theta=1$, that is, a backward scheme taking stress at the end of the increment, normally lower, leads to an under-prediction of the viscoplastic strain, with shear stresses decreasing more slowly.

A second calculation has been run with $c=10.0 \mathrm{kN} / \mathrm{m}^{2}$. In this case, the analytical solution is not straightforward, and the numerical solution obtained for very small steps of $\Delta t=0.001 \mathrm{~s}$, which leads to practically the same results for any $\theta$, is taken as the 'exact' solution. This 'exact' solution is represented in Figure 3 together with the numerical results obtained with $\theta=0, \theta=1 / 2$, and $\theta=1$, first for $\Delta t=1 \mathrm{~s}$ (left diagram) and then for $\Delta t=2.0 \mathrm{~s}$ and $\Delta t=0.5 \mathrm{~s}$ (right diagram).

Figure 4 (left) shows a similar trend as Figure 3. Figure 4 (right) shows some additional features of the integration schemes, confirming the better performance of $\theta=1 / 2$ and showing that for larger time increments ( $\Delta t=2 \mathrm{~s}$ ), convergence is not even obtained for $\theta=0$. The overall error of the three solutions in terms of area under the curves for $\Delta t=0.5 \mathrm{~s}$ are less than $0.1 \%$ for $\theta=1 / 2$ in front of $4.4 \%$ and $4.3 \%$ for $\theta=0$ and $\theta=1$, respectively. Additionally, calculations have been also run for $\Delta t=0.2 \mathrm{~s}$ with errors of $1.7 \%$ for both $\theta=0$ and $\theta=1$. And one has to decrease $\Delta t$ to the value of $\Delta t=0.05 \mathrm{~s}$ to obtain errors of less than $1 \%$ for those values of $\theta$. This means that for those strategies $(\theta=0$ and $\theta=1)$, about 20 times more time steps are required to obtain similar accuracy as for $\theta=1 / 2$. Also remarkable is the observation that the backward scheme $\theta=1$ 


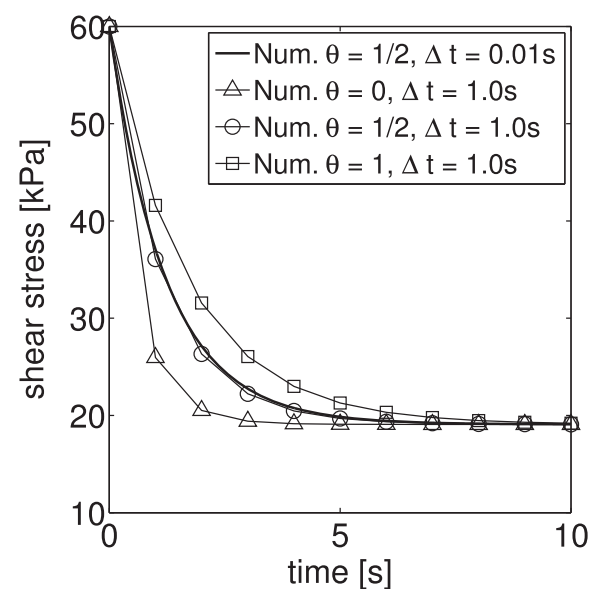

(a)

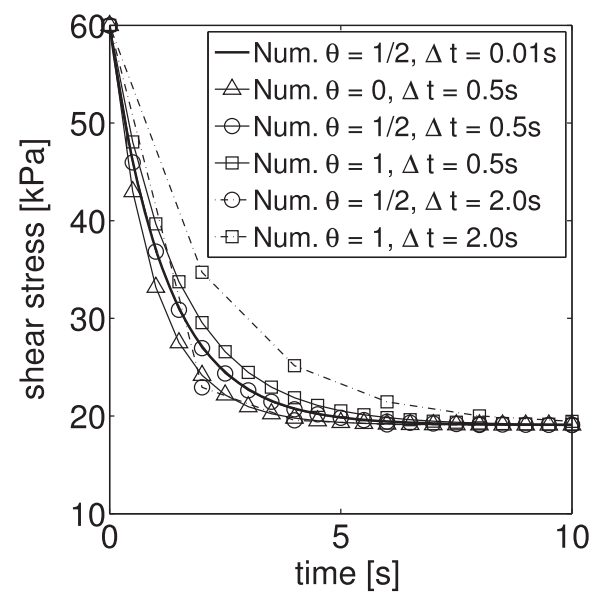

(b)

Figure 4. (a) Numerical results for the evolution of the shear stress with time for $\Delta t=1 \mathrm{~s}$ and (b) $\Delta t=0.5 \mathrm{~s}$ and $\Delta t=2 \mathrm{~s}$.

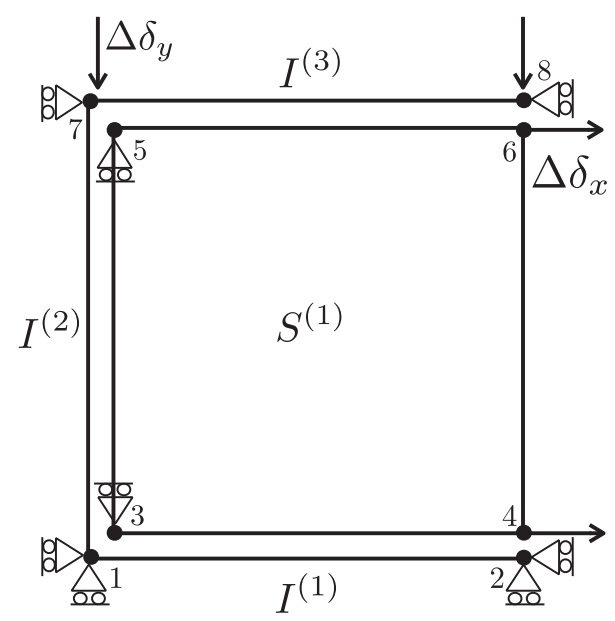

Figure 5. Pull-out test. FE mesh and boundary conditions.

(usually assumed in strain-driven cases to be a preferred choice) in this context of stress-driven scheme, and at least in this example, leads to similar errors as the forward scheme with $\theta=0$.

5.2.2. Semi-constitutive pull-out test, solved using viscoplastic relaxation. This example consists of a pull-out test with the following setup: a quadrilateral $(2 \mathrm{~m} \times 2 \mathrm{~m})$ continuum element $\left(S^{(1)}\right)$ surrounded by three zero-thickness interface elements $\left(I^{(1)}, I^{(2)}, I^{(3)}\right)$, as shown in Figure 5 . The analysis is carried out using the VPR procedure with both fixed $\Delta t$ and variable $\Delta t$ and then automatic sub-stepping, and then, the performance of both algorithms (with $\theta=1 / 2$ ) is analyzed and compared.

As it can be seen in Figure 5, the horizontal nodal forces on nodes 5 and 3 collect shear contributions from interfaces $I^{(1)}$ and $I^{(3)}$, as well as tensile contribution from $I^{(2)}$. Results from this example may also help verifying that the procedure is reliable when the viscoplastic strain rate differs in orders of magnitude for different Gauss points, as is the case for the upper and lower interfaces, which will slide in shear/compression, in contrast to the left interface which will open in tension.

Two loading steps are considered in this pull-out test. In the first one, a vertical displacement increment $\left(\Delta \delta_{y}\right)$ is imposed to the top nodes in order to generate a state of normal compression at the 
top and bottom perimetral interfaces. In the second step, the horizontal displacement is prescribed incrementally to nodes 4 and 6 in order to pull the quadrilateral element out of the surrounding interfaces. Each pull-out displacement increment $\left(\Delta \delta_{x}\right)$ is, in turn, divided in two sub-increments. In the first one, the displacement $\Delta \delta_{x}$ is applied instantaneously (with $\Delta t=0$ ), and in the second, time is allowed to pass $(\Delta t>0)$ at constant displacement $\left(\Delta \delta_{x}=0\right)$. Because of the viscoplastic behavior of the three perimetral interfaces, the stress increments generated in the 'instantaneous' pull-out sub-increment will then relax during the second sub-increment. For this particular case, the vertical displacement increment imposed at the first step was $\Delta \delta_{y}=-3.4641 \cdot 10^{-4} \mathrm{~m}$, which generated a compressive normal stress of $\sigma_{n}=-98.0397 \mathrm{kPa}$, and each horizontal displacement increment imposed was $\Delta \delta_{x}=1.7081 \cdot 10^{-5} \mathrm{~m}$.

As stated, the problem only exhibits two unknown degrees of freedom that correspond to the horizontal displacements of nodes 5 and 3, because all other displacements are prescribed. This leads to a drastic simplification of the problem, if compared with the corresponding full matrix system within the FE framework, and it becomes possible to reach a semi-analytical solution as shown in Appendix B.

For the numerical calculations, the continuum is considered linear elastic (plain strain with $E=$ $6 \cdot 10^{5} \mathrm{kPa}$ and $v=0$ ), and the interfaces are viscoplastic with the following parameters: $K_{N}=$ $K_{T}=10^{7} \mathrm{kPa} / \mathrm{m}, c=10 \mathrm{kPa}, \tan \phi=1.4$, and $\eta=6 \cdot 10^{5} \mathrm{kPa} \mathrm{s}, F_{0}=28.0$.

For the purpose of comparison of the proposed scheme with variable pseudo time step, some additional calculations have been run with fixed step, first with very large number of time increments of very small size ('exact' calculation) and then with fewer steps of much larger size. Some calculations were also performed using different values of the parameters that control the VPR scheme previously presented. All these results will be compared with the 'exact' solution obtained with the fixed step ( $\Delta t=2.5 \mathrm{~s}$ and 2000 increments). According to the symmetry, only the upper part of the geometry is considered for the representation of the results. The plots of stressrelative displacement of the Gauss points that are at the same geometric position as nodes 5 and 3 are considered. In addition, because of the loading conditions, only the normal stress-normal relative displacement of the Gauss point belonging to the interface $I^{(2)}$ and the shear stresstangential relative displacement of the Gauss point belonging to $I^{(3)}$ are represented, as shown in both Figures 6 and 7.

Firstly, VPR with a fixed iteration $\Delta t$ is taken into account (Figure 6). This case can be considered as the simplest scheme for VPR. In particular, four different values of $\Delta t$ were used: 2.5, 10.0, 20.0 , and $40.0 \mathrm{~s}$, as can be observed in Figure 6 . The number of iterations that were needed to reach convergence was $2454,613,307$, and 154 , respectively. A tolerance of $10^{-5}$ was used for the nor-

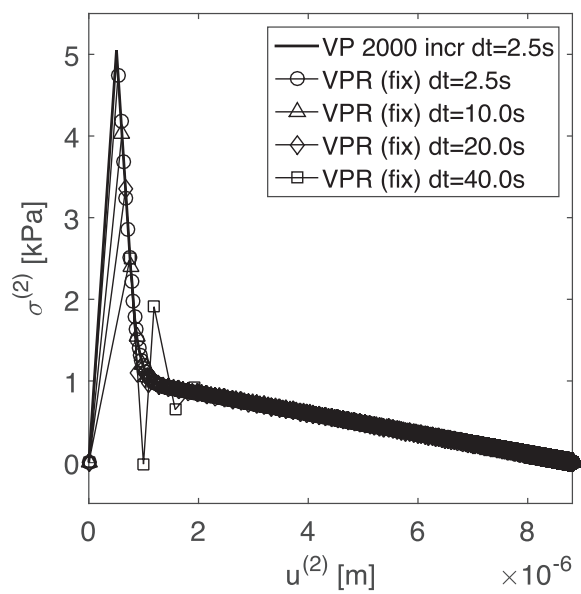

(a) Normal stress - Normal relative displacement

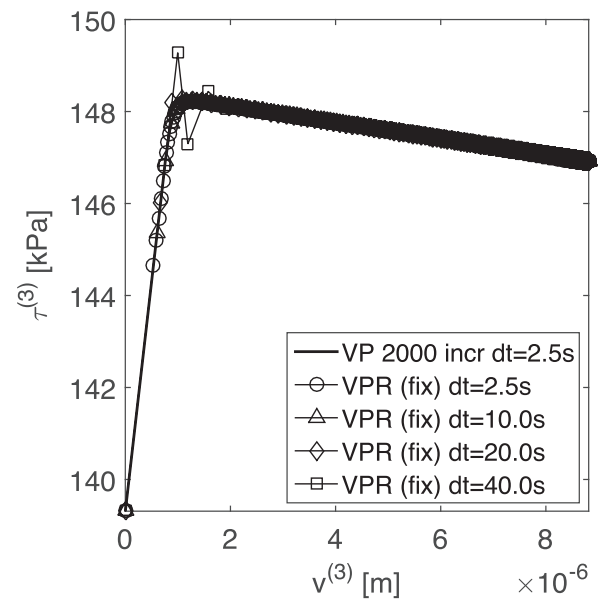

(b) Shear stress - Tangential relative displacement

Figure 6. Stress-displacement evolution curves obtained with physical viscoplasticity with 2000 incr. and $\Delta t=2.5 \mathrm{~s}$, and with the viscoplastic relaxation procedure with $\Delta t$ fixed and equal to $2.5,10.0,20.0$, and 40.0 . 


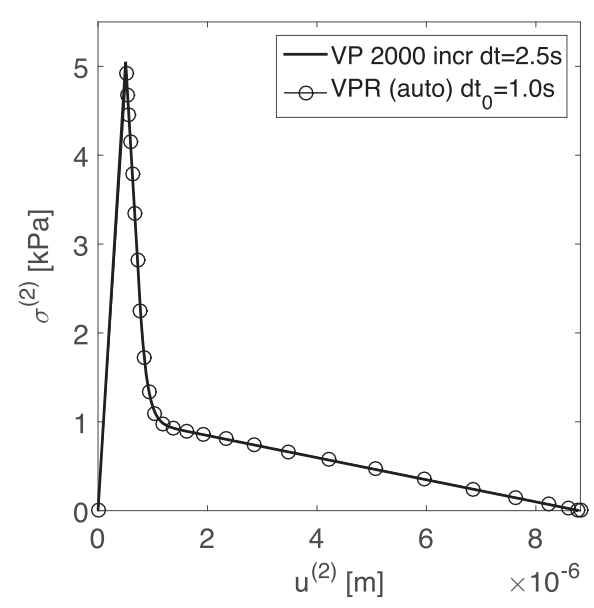

(a) Normal stress - Normal relative displacement

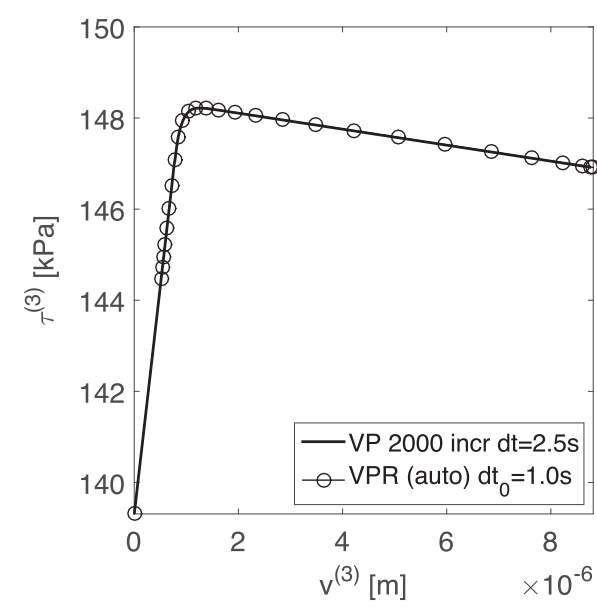

(b) Shear stress - Tangential relative displacement

Figure 7. Stress-displacement evolution curves obtained with physical viscoplasticity with 2000 incr. and $\Delta t=2.5 \mathrm{~s}$ and with the viscoplastic relaxation procedure with automatic control of $\Delta t$.

malized residual force at node 5. Although the number of iterations is smaller than the total amount of iterations needed to obtain the evolution curve with physical viscoplasticity (2000 increments and an average of 2 iterations per increment), those values are still notably high. Moreover, because the slope of the curve for small values of the displacements is significant, when the value of $\Delta t$ is too large instability can occur. In this example, for the value of $\Delta t=40.0 \mathrm{~s}$, even though the procedure seems to be unstable in the first four iterations, at the end of the iterations, the same result is obtained.

Another aspect that can be noticed in Figure 6 is that there is a change of slope in the evolution curve. Because the slope decreases significantly and the stress state gets close to the yield surface, even if the value of $\Delta t$ is large, an important amount of iterations is needed. The VPR scheme implemented allows an automatic scaling of the time increment in the iterations that can be very advantageous in these situations. As it was detailed previously, the automatic control takes into account the difference of the viscoplastic strain increments obtained a priori and a posteriori in the iteration. In this particular example, the value of $\Delta t$ will be kept small at the beginning and during the change of slope, but it will be increased eventually when the slope becomes constant.

As shown in Figure 7, using the VPR scheme with automatic control of time increment, only 30 iterations were needed to reach convergence. The initial value of the time increment was $\Delta t=1.0 \mathrm{~s}$, the factor used to increase $\Delta t$ was 1.35 , and the tolerance value used to increase the time increment was $10^{-5}$. The value of $\Delta t$ in the first 10 iterations remained close to $10 \mathrm{~s}$ and then increased to a maximum value of $2447.25 \mathrm{~s}$ in the last iteration.

5.2.3. Wedge failure mechanism with prescribed displacement rate. In this example, the symmetric part of a 3D wedge failure mechanism is analyzed (Figure 8). The wedge is formed by the intersection of two planes sitting on the line contained in the symmetry plane (line $l_{c h}$ ) and dipping $45^{\circ}$ (angle $i$ ) and on horizontal lines with directions (strikes) $45^{\circ}$ and $135^{\circ}$ with respect to to the $x$-axis. This results in a dip angle $\beta$ for those planes of $54.74^{\circ}$, as also represented in Figure 8 .

The horizontal displacement along the $x$-direction is prescribed to zero value for all the nodes over the symmetry plane $(x=0)$, and so they are the vertical $(z)$ displacements at the base of the specimen $z=0$ and the 'forward' $(y)$ displacements of the rear side $(y=0)$ of the lower part of the specimen. The material properties of the interface are $K_{N}=K_{T}=10^{8} \mathrm{kPa} / \mathrm{m}, c=10 \mathrm{kPa}$, $\phi=30^{\circ}$, and $\eta=10^{4} \mathrm{kPa}$ and the continuum: $E=5 \cdot 10^{6} \mathrm{kPa}$ and $v=0.2$.

In this numerical example, physical VP and VPR (fixed $\Delta t$ ) schemes with $\theta=0.5$ are taken into account. Loading consists of uniform prescribed vertical $(z)$ displacements at the top of the specimen, while horizontal $(x, y)$ displacements at the same nodes are left free. In the physical VP cases, the prescribed vertical displacement was applied in small increments of $\Delta t=0.1 \mathrm{~s}$ from the beginning of the test. While in the VPR cases, two loading steps are applied: (1) A first 'elastic' 

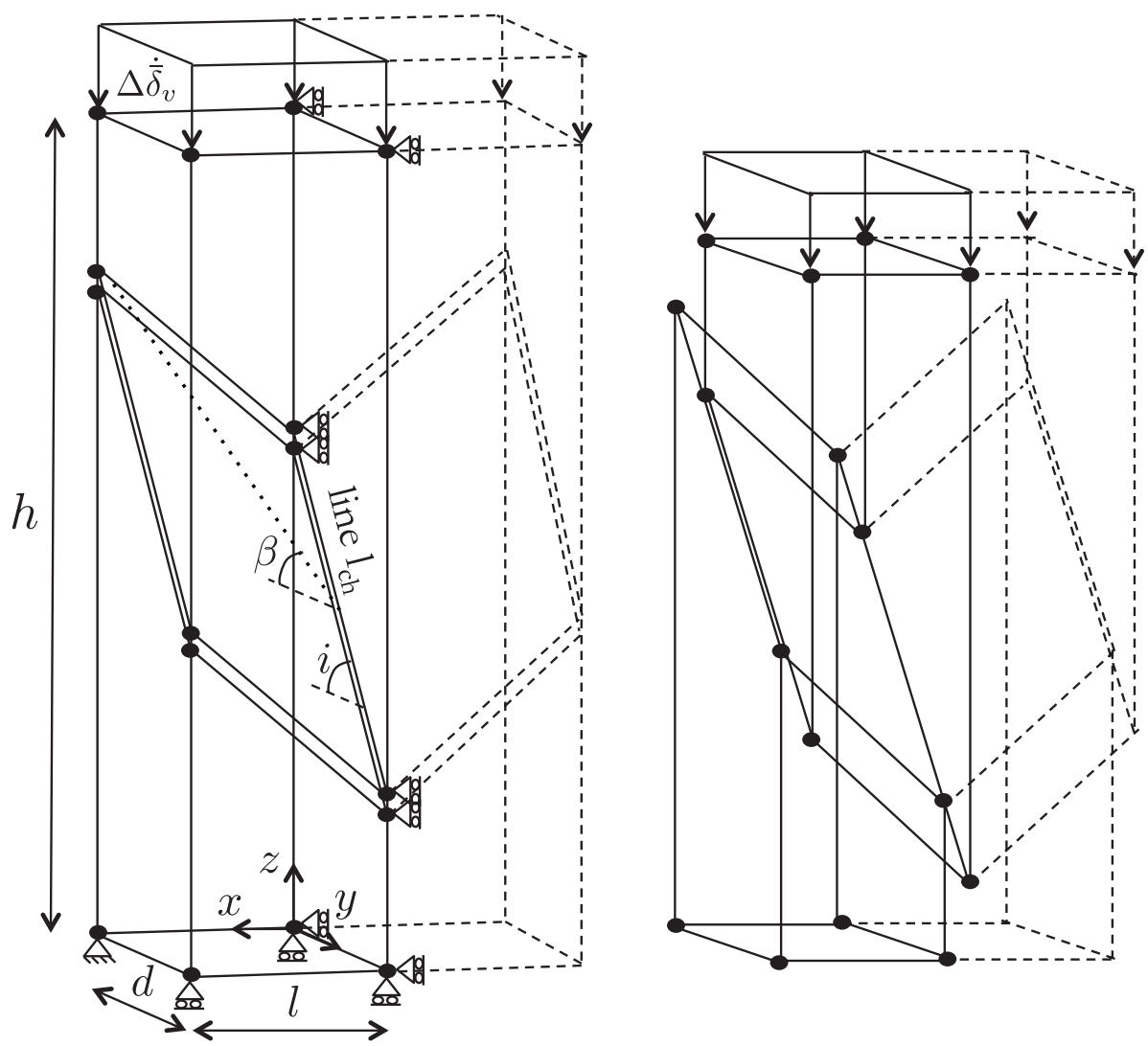

Figure 8 . Geometry of the wedge failure mechanism symmetric part (left) and deformed mesh $(\times 2000)$ at the end of the second step (right). The volume of solid containing the wedge has the dimensions: $h=2.5$, $d=1.0$ and $l=0.5 \mathrm{~m}$.

increment $\left(\Delta \delta_{v}=-5 \cdot 10^{-5}\right.$ and $\left.\Delta t=0 \mathrm{~s}\right)$ which leads to a uniaxial stress state $\left(\sigma_{y y}=-98.8585\right.$ $\mathrm{kPa}$ ). The stress state in terms of normal and shear stresses on the plane is obtained by static equilibrium. This initial stress state is such that the shear stress vector is oriented in the direction of the maximum dip direction of the plane (dotted line in Figure 8). It also should be noticed that stress states that satisfy this static equilibrium are represented by a fixed relationship between normal and shear stresses: $\sigma / \tau=\tan \beta$, as seen in Figure 10 (bottom); (2) this is followed by relaxation increments by applying the prescribed displacement rate at the upper boundary (variable increments of $\Delta \delta_{v}$ with $\Delta t=0.1 \mathrm{~s}$ ). From that point on, a more complex stress state will develop, with growing horizontal stress components in the wedge that will progressively deviate the shear traction on the plane until it reaches asymptotically the direction also contained in the plane but parallel to the symmetry plane defined by line $l_{c h}$ (only kinematically admissible failure mechanism).

The prescribed vertical displacement has been applied progressively, at various (constant) strain rates, ranging from an infinitely slow rate (equivalent to perfect inviscid elasto-plasticity) to a higher strain rate of $2.5 \cdot 10^{-6} \mathrm{~ms}^{-1}$. The results of the case are depicted in Figure 9, where the physical VP simulations appear as light lines while VPR as bold lines. Physical VP vertical reaction monotonically increases with the vertical displacement, while VPR cases show an initial elastic response, followed by a nonlinear curve (as the various integration points of the interface are progressively reaching the plastic state), and finally a plateau (when all integration points become plastic). Note that the load value of the plateau depends on the strain rate, and for the same strain rate, both physical VP and VPR cases reach the same vertical reaction value. This value can be obtained analytically as shown later on.

Because the wedge failure mechanism is kinematically admissible and the loading conditions lead to stress states (Figures 10 and 11) beyond the yield surface, viscoplastic shear strains develop, and 
the wedge slides in the direction of the intersection because of the symmetry of the problem. Considering the kinematic relationship between the prescribed displacement rate (vertical direction) and the viscoplastic shear strain rate developed during sliding ( $i$-direction), the following relationship exists:

$$
\Delta \dot{\delta}_{\tau}^{v p}=\frac{\Delta \dot{\bar{\delta}}_{v}}{\sin i}=\frac{1}{\eta} \frac{F(\sigma)}{F_{0}}
$$

According to this previous expression, it is possible to determine a surface $\bar{F}$ representing the stress states in failure stationary conditions, when stress redistribution at structure level is not possible. This surface is therefore related to the prescribed displacement rate value according to the following expression:

$$
\bar{F}=F-\eta F_{0} \dot{\bar{\delta}}_{v} \frac{1}{\sin i}=0
$$

In this example, besides the infinitely slow case $\left(\Delta \dot{\bar{\delta}}_{v 0}=0.0\right)$, three different values of the prescribed displacement rate were considered $\left(\Delta \dot{\bar{\delta}}_{v 1}=2.5 \cdot 10^{-6} \mathrm{~ms}^{-1}, \Delta \dot{\bar{\delta}}_{v 2}=10^{-6} \mathrm{~ms}^{-1}\right.$, $\Delta \dot{\bar{\delta}}_{v 3}=0.5 \cdot 10^{-6} \mathrm{~ms}^{-1}$ ), which were applied in the following: 800 physical VP and $600 \mathrm{VPR}$; 2000 physical VP and $1500 \mathrm{VPR}$; and 4000 physical VP and $3000 \mathrm{VPR}$ increments of $\Delta t=0.1 \mathrm{~s}$, respectively, in order to reach the same amount of imposed vertical displacement at the end of the test (Figure 9). In terms of the number of iterations, the cases computed with physical VP need two iterations per time increment, resulting in 1600, 4000, and 8000 iterations, respectively. However, the VPR algorithm requires three to five iterations during the first 20 increments and two iterations the remaining of the test, which yields the total number of iterations of 1225,3025 , and 6026, respectively. The deformed mesh at the end of the test is shown in Figure 8. All the background for this example summarized in the previous paragraphs is shown in Figures 9-11 and discussed as follows.

Figure 9 illustrates the force-displacement evolution during the test until a steady state is reached at failure. For each case, the evolution curve can be divided in three parts. In the first part, which corresponds to step 1, the behavior is elastic (linear slope) because the vertical displacement incre-

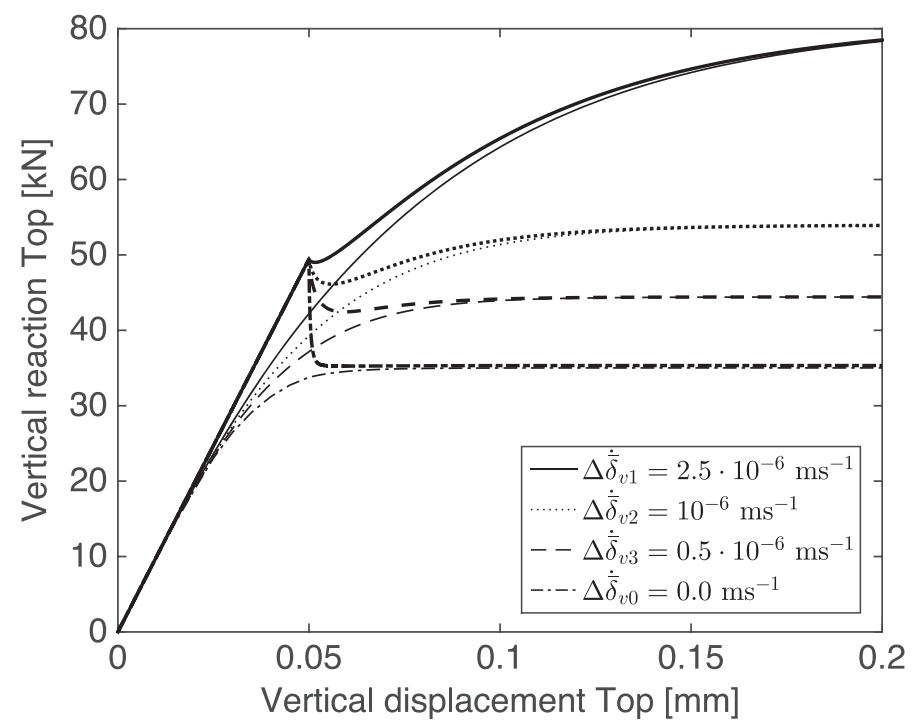

Figure 9. Load-displacement evolution curves obtained with physical VP (light lines) and VPR (bold lines), vertical displacement rates equal to $0.0,0.5 \cdot 10^{-6}, 10^{-6}$ and $2.5 \cdot 10^{-6} \mathrm{~ms}^{-1}$. 

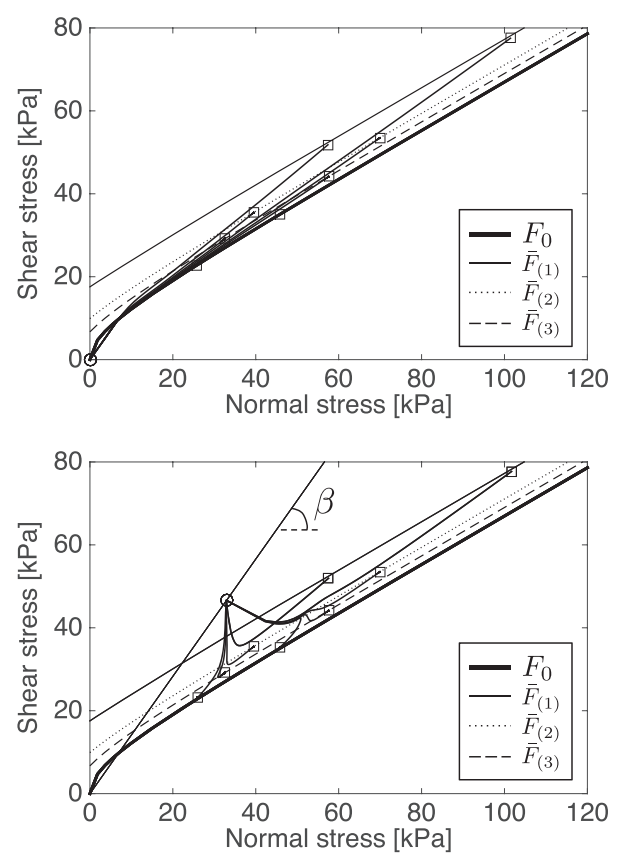

Figure 10. Stress path in the $\sigma-\tau$ plane for vertical displacement rates equal to $0.0,0.5 \cdot 10^{-6}, 10^{-6}$ and 2.5 . $10^{-6} \mathrm{~ms}^{-1}$ Top: physical VP Bottom: VPR.
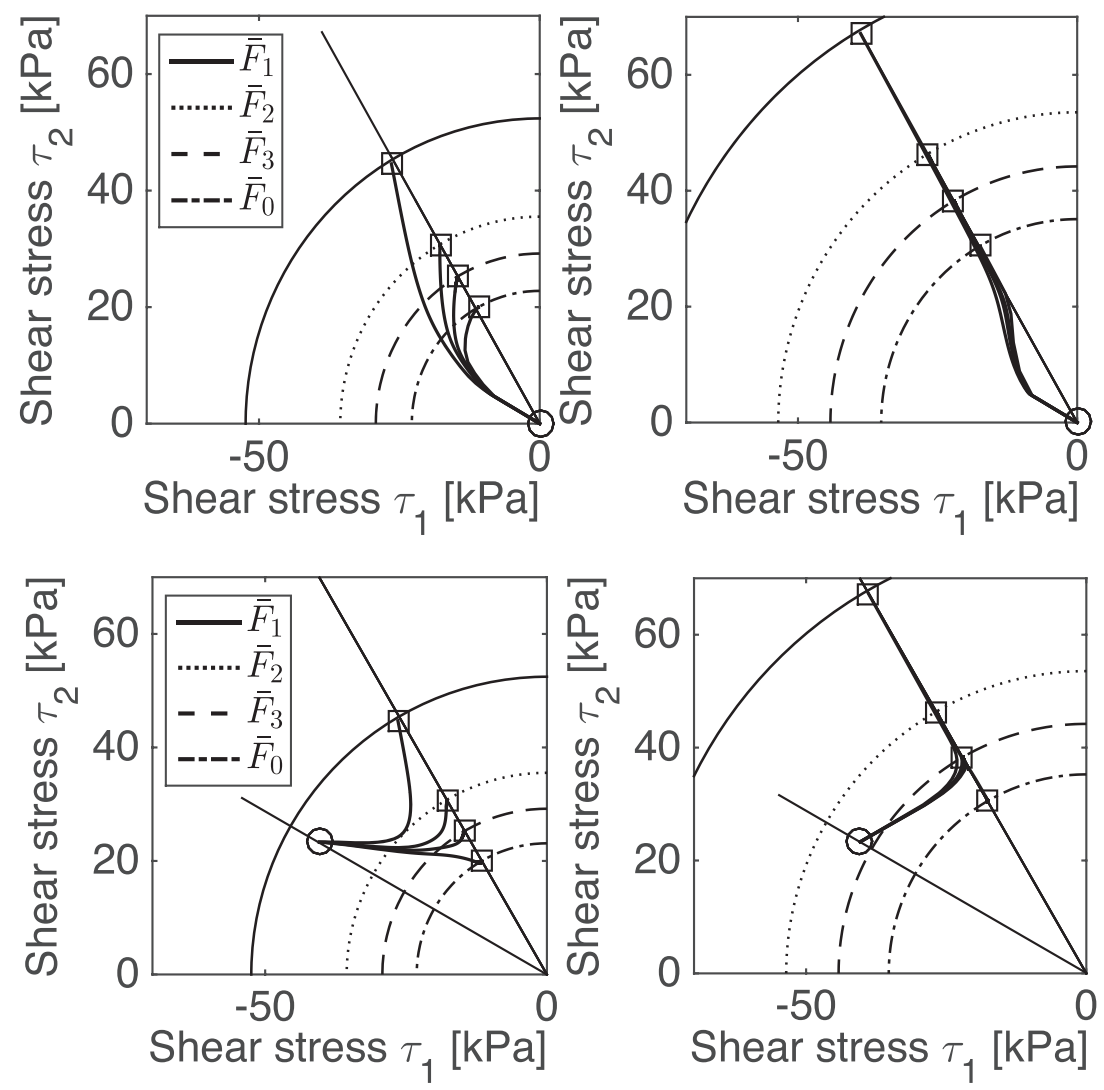

Figure 11. Stress path in the $\tau_{1}-\tau_{2}$ plane for vertical displacement rates equal to $0.0,0.5$. $10^{-6}, 10^{-6}$ and $2.5 \cdot 10^{-6} \mathrm{~ms}^{-1}$. Left: outer integration points; right: inner integration points; top: physical VP; bottom: VPR. 
ment is applied at $\Delta t=0 \mathrm{~s}$. Though not very significant, this elastic part also exists in the physical VP cases because, initially, the stress path develops inside the yield surface; see Figure 10 (top). During the second and third parts, both included in step 2, the behavior is viscoplastic, and sliding due to shear viscoplastic strains occurs. Reorientation of the shear stresses takes place in the second part while the steady state conditions develop in the third part of the curve. In this last part, it can be observed that the VPR curves reach the inviscid solution (physical VP curves).

As commented previously, this example consists of a wedge failure discretized in two solid hexahedra elements and a quadrangular interface element. A Newton-Cotes quadrature of four integration points at the corner nodes of the quadrangle was used to integrate in space. It is important to notice that two integration points are located at the intersection line while the other two are located at the outer boundary. Because of the symmetry of the problem, the same stress state is obtained for each pair of integration points. For this reason, only two stress paths for each case are plotted in Figures 10 and 11. Note that in Figure 11, the straight solid line denotes the direction of the intersection line in terms of $\tau_{2} / \tau_{1}$.

Figure 10 shows the stress paths in $\sigma-\tau$ plane $\left(\tau=\sqrt{\tau_{1}^{2}+\tau_{2}^{2}}\right)$ while Figure 11 shows the stress paths in $\tau_{1}-\tau_{2}$ plane. In both figures, the initial stress state is represented as a circle (after step 1 for VPR cases), while the final state at failure for each integration point is represented as a square. Also in both plots, the initial yield surface $\left(F_{0}\right)$ and the final surfaces $(\bar{F})$ given by $(20)$ are represented. As it can be noticed in both plots and for both physical VP and VPR cases, the initial stress state evolves in the sense that normal stresses increase and shear stresses change their orientation in order to align to the intersection line. Note that less viscoplastic strain is needed for the reorientation of the integration points that are located at the intersection line (Figure 11, right). When the steady state is reached at failure, stresses reach the corresponding $\bar{F}$ surfaces according to the imposed value of vertical displacement rate. As it has also been stated for Figure 9, although the stress paths are different for physical VP and VPR (top and bottom plots in Figures 10 and 11), the final stress states are the same for a given vertical strain rate.

\section{CONCLUDING REMARKS}

Stress-prescribed schemes seem very advantageous for integrating Perzyna-type viscoplasticiy, because the constitutive evaluation is reduced to the numerical evaluation of an integral. Based on this idea, the general scheme described allows to recover as limit cases, both the original Zienckiewicz scheme with constant stiffness and a classical backward Euler formula. By proper linearization of the variation of the loading function and some additional simplifications, the scheme presented also leads to a simple form of the tangent compliance that can be inverted in closed-form to obtain explicitly the so-called m-AGC tangent operator. This operator is shown to perform very satisfactory in the examples analyzed.

The constitutive schemes developed can also be applied in the context of VPR, in which case, instead of iterations, the pseudo-time-step size is readjusted till the m-AGC tangent provides sufficiently good prediction without iteration.

The performance of both schemes (physical viscoplasticity and VPR) has been shown in the case of three examples of application. In the context of a constitutive relaxation test (physical VP scheme), analyzing the shear stress decay with time, which can be obtained in closed-form, the strategy with $\theta=1 / 2$ appears to be more accurate than $\theta=0$ and 1 , obtaining the error as the difference of areas under the numerical and analytical curves. Other examples in the FEs framework point out the capacity of the proposed schemes to capture stress paths derived from more complex load conditions, in particular, combined shear sliding/tensile opening and stress reorientation while sliding. It is also worth to mention the good performance of the proposed VPR algorithm, in terms of the number of iterations, because the (pseudo) time-step can be automatically scaled during the iterative procedure. 


\section{APPENDIX A: STRESS-DRIVEN VS STRAIN-DRIVEN STRATEGIES}

In this appendix, both stress-driven (A.1) and strain-driven (A.2) integration strategies are described. Also, an application example at constitutive level is provided for comparison purposes.
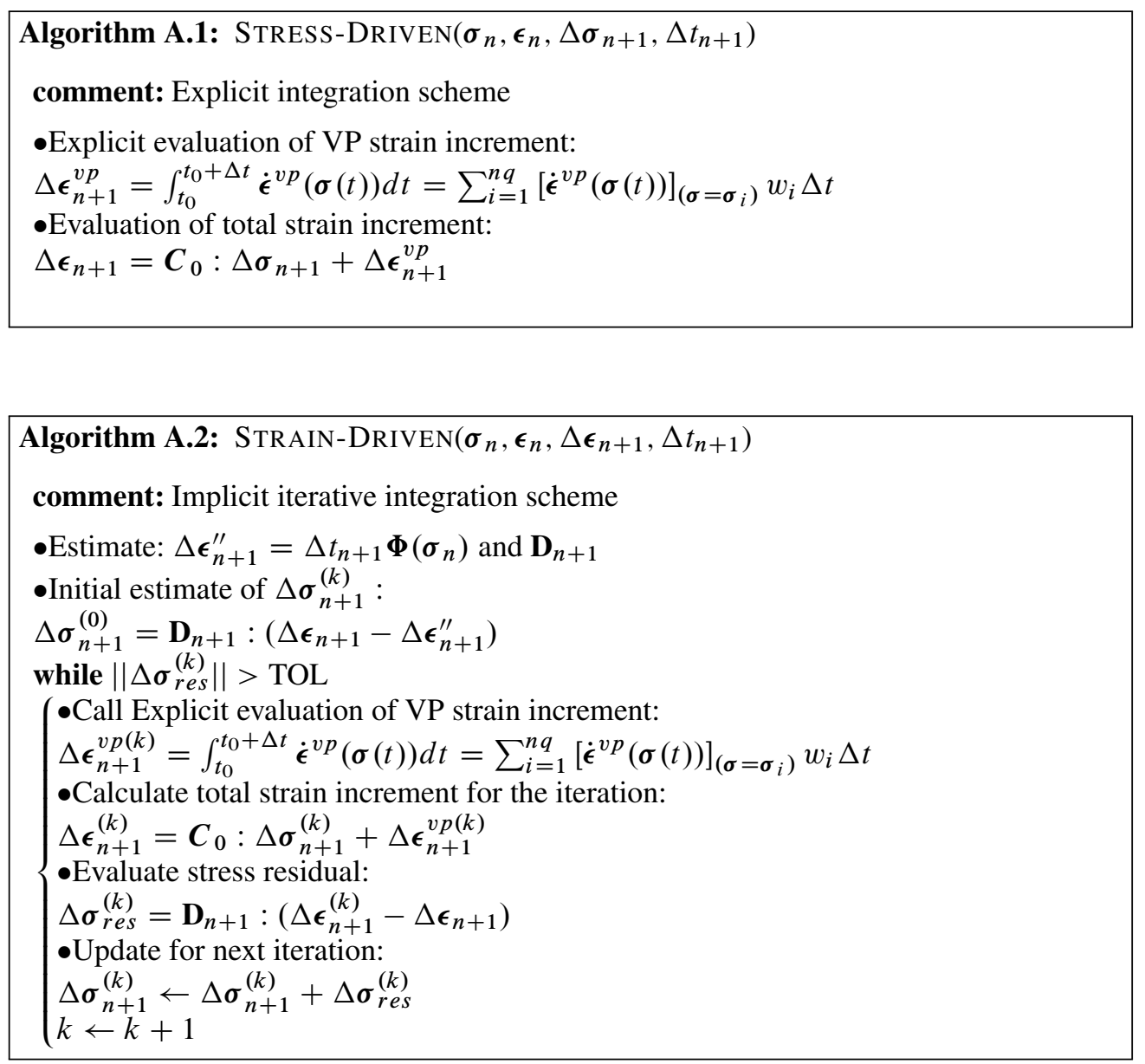

In order to run a valid illustration example of the aforementioned comparison, one needs an increment of stress applied during a specific time increment and the corresponding increment of strain. This may be most conveniently extracted from a Gauss point of a converged finite element calculation. For this purpose, the simplest possible two-dimensional failure geometry has been considered, which consists of a rectangular specimen with an embedded plane dipping $45^{\circ}$ (Figure A1). This plane has been discretized using a single interface element $\left(K_{N}=K_{T}=10^{7} \mathrm{kPa} / \mathrm{m}, c=10 \mathrm{kPa}\right.$, $\tan \phi=0.577$, and $\left.\eta=6 \cdot 10^{5} \mathrm{kPa} \mathrm{s}\right)$.

In the first step, an instantaneous $(\Delta t=0)$ vertical displacement of $\Delta \delta_{y}=-2.6667 \cdot 10^{-4} \mathrm{~m}$ is prescribed to the upper boundary of the specimen, which leads to an initial state of $\sigma_{0}=$ $(-39.169097,39.169097)$ and $\epsilon_{0}=\left(-3.9169097 \cdot 10^{-6}, 3.9169097 \cdot 10^{-6}\right)$.

As a second step, a time increment of $\Delta t=0.25 \mathrm{~s}$ has been calculated using the finite element code, and the following converged state has been obtained: $\sigma_{1}=(-39.0318847,39.0318847)$ and $\boldsymbol{\epsilon}_{1}=\left(-3.0318847 \cdot 10^{-6}, 5.22428045 \cdot 10^{-6}\right)$. This solution is taken as the input for the comparison itself, consisting of

1. The stress-driven algorithm is used first to integrate from the initial state $\left(\sigma_{0}, \epsilon_{0}\right)$ a stress increment of $\Delta \sigma_{1}=(0.1372123,-0.1372123)$ applied during a $\Delta t=0.25 \mathrm{~s}$ time increment, which results in $\epsilon_{1}=\left(-3.90318847 \cdot 10^{-6}, 5.22428044 \cdot 10^{-6}\right)$, that is, a direct explicit calculation with results practically identical to the converged $\mathrm{FE}$ value. 


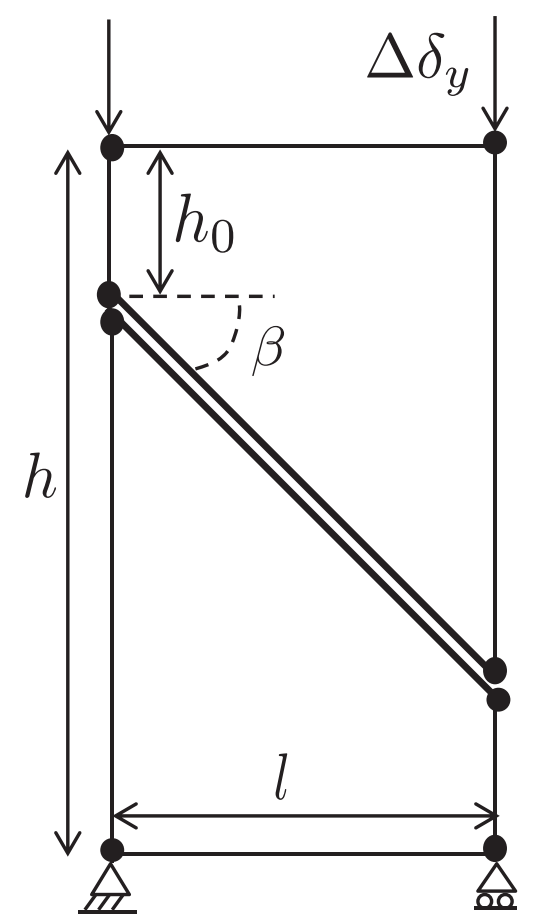

Figure A1. Two-dimensional specimen with inclined interface $\left(\beta=45^{\circ}\right) . l=1 \mathrm{~m}, h=2 l, h_{0}=l / 2$, and $\Delta \delta_{y}=-2.6667 \cdot 10^{-4} \mathrm{~m}$ have been considered in this example.

Table A1. Stress-driven implicit integration results.

\begin{tabular}{ccc}
\hline & $\mathbf{D}_{0}$ & $\mathbf{D}_{n+1}$ \\
\hline TOL $=10^{-3}$ & $\sigma_{1}=(-39.0318847,39.0296576)$ & $\sigma_{1}=(-39.0318847,39.0330322)$ \\
& 639 iters & 5 iters \\
TOL $=10^{-5}$ & $\sigma_{1}=(-39.0318847,39.0319071)$ & $\sigma_{1}=(-39.0318847,39.0318942)$ \\
& 1004 iters & 7 iters \\
TOL $=10^{-7}$ & $\sigma_{1}=(-39.0318847,39.0318845)$ & $\sigma_{1}=(-39.0318847,39.0318848)$ \\
\hline
\end{tabular}

2. The strain-driven algorithm is finally applied with the initial state and prescribed $\Delta \boldsymbol{\epsilon}=$ $\left(1.372123 \cdot 10^{-8}, 1.307370747 \cdot 10^{-6}\right)$ and $\Delta t=0.25$. The results that are shown in Table A1, on top of not being explicit and requiring iterations, turn out strongly dependent of tolerance and stiffness.

\section{APPENDIX B: SEMI-CONSTITUTIVE PULL-OUT TEST EQUATIONS}

The classic FEM structure $\boldsymbol{f}=\boldsymbol{K} \boldsymbol{\delta}$, where $\boldsymbol{f}$ and $\boldsymbol{\delta}$ are the global nodal force and nodal displacement vectors, respectively, and $\boldsymbol{K}$ is the global stiffness matrix, can be simplified in this particular example. In Figure B1, the contribution of the solid and interface elements to the global stiffness matrix is represented, from which an expression of the horizontal force in the node $5\left(f_{5}^{x}\right)$ can be obtained as follows:

$$
f_{5}^{x}=\sum_{i=1}^{n n o d e}\left(K_{5 i}^{(x x)} \delta_{i}^{x}+K_{5 i}^{(x y)} \delta_{i}^{y}\right)
$$




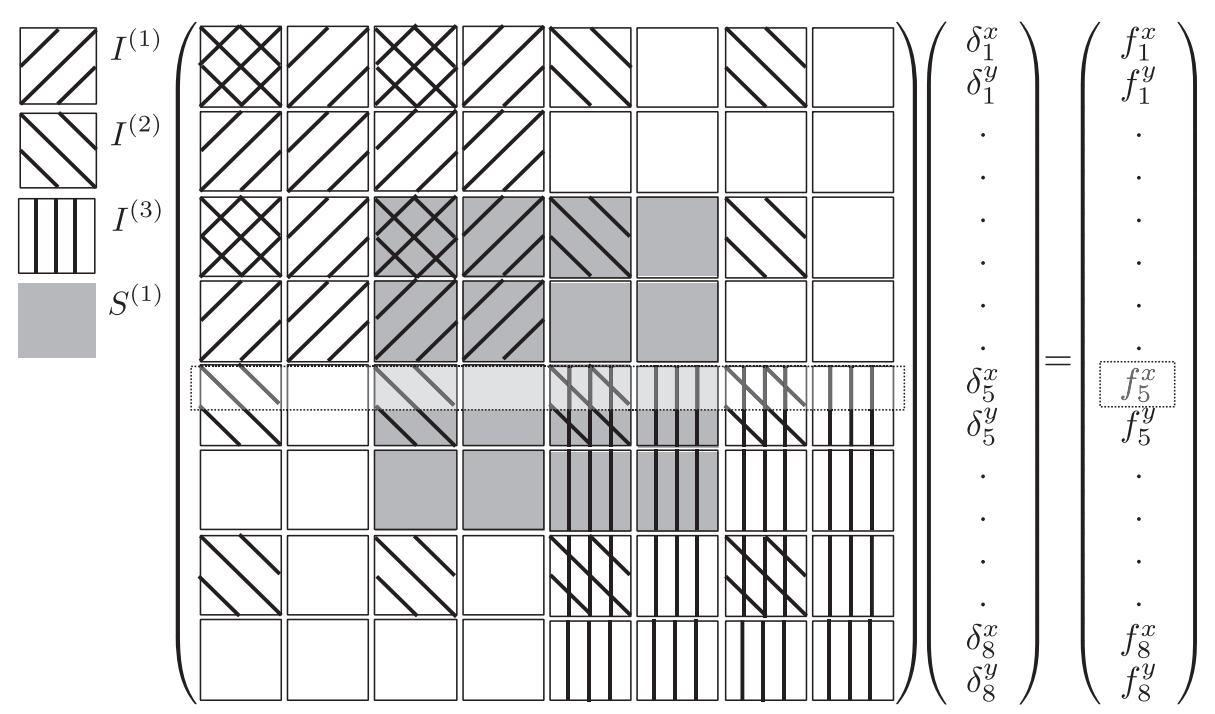

Figure B1. Continuum and interface elements contribution to the global stiffness matrix.

Because $\delta_{3}^{x}$ and $\delta_{5}^{x}$ are the only degrees of freedom and they have both the same value according to the symmetry of the problem, the previous equation reduces to

$$
f_{5}^{x}=K_{55}^{(x x)} \delta_{5}^{x}
$$

Therefore, a scalar relationship is stablished between the horizontal force and horizontal displacement of node 5 , by the component $K_{55}^{(x x)}$ of the global stiffness matrix. As it can be observed in Figure B1, this component has the contribution of two interface elements $\left(I^{(2)}\right.$ and $\left.I^{(3)}\right)$ stiffness and the continuum element stiffness. The interface $I^{(2)}$ contributes in terms of normal stiffness while the interface $I^{(3)}$ in terms of transversal stiffness.

$$
K_{55}^{x x}=K_{N}^{I^{(2)}}+K_{T}^{I^{(3)}}+K^{S^{(1)}}
$$

where $K^{S^{(1)}}$ stands for the stiffness contribution of the fourth local node of the continuum element $S^{(1)}$. Considering plain strain elasticity and $v=0$, for the sake of simplicity, it can be obtained as follows:

$$
K^{S^{(1)}}=\int_{-1}^{+1} \int_{-1}^{+1}\left[\begin{array}{lll}
\frac{\partial N_{4}}{\partial x} & 0 & \frac{\partial N_{4}}{\partial y}
\end{array}\right]\left[\begin{array}{ccc}
E & 0 & 0 \\
0 & E & 0 \\
0 & 0 & E / 2
\end{array}\right]\left[\begin{array}{c}
\frac{\partial N_{4}}{\partial x} \\
0 \\
\frac{\partial N_{4}}{\partial y}
\end{array}\right] d \eta d \xi=\frac{E}{2}
$$

The iterative procedure takes into account the stress redistribution, associated to the viscoplastic strains, at the interfaces $I^{(2)}$ and $I^{(3)}$. At $I^{(2)}$, a tensile residual stress is produced while at $I^{(3)}$ the residual stress only presents the shear component. This residual stresses are then integrated, and the nodal force increment is obtained and assembled to $f_{5}^{x}$. According to the geometry of this example, the contributive area to the calculation of the nodal forces is unitary, and the integration is simple; leading to the increments of forces are the increment of stresses. The simplified VPR iterative scheme is summarized in the Algorithm B.1. 


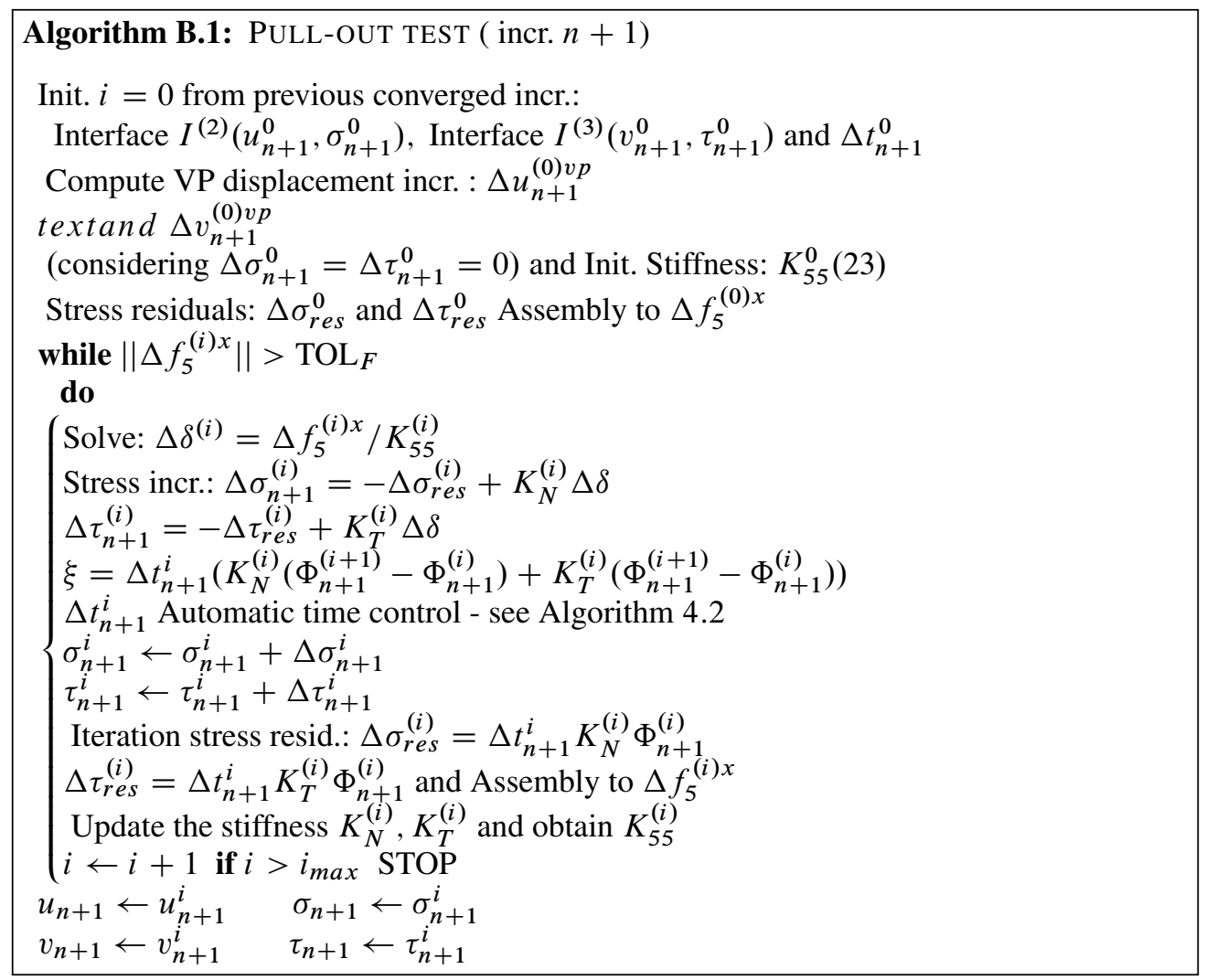

\section{ACKNOWLEDGEMENTS}

Partial support for this research has come from project BIA-2012 36898 MEC (Madrid), which includes FEDER funds (European Comission). Funding from 2009SGR-180 from Generalitat de Catalunya (Barcelona) is also greatly appreciated. The first author also wishes to thank MEC (Madrid) for the doctoral fellowship including funds for a 6-month stay at CU-Boulder.

\section{REFERENCES}

1. Zienkiewicz OC, Cormeau IC. Visco-plasticity - plasticity and creep in elastic solids - a unified numerical solution approach. International Journal for Numerical Methods in Engineering 1974; 8(4):821-845.

2. Cormeau I. Numerical stability in quasi-static elasto/visco-plasticity. International Journal for Numerical Methods in Engineering 1975; 9(1):109-127.

3. Underwood P. Dynamic Relaxation, Computational Methods for Transient Analysis, Vol. 1. Elsevier: Amsterdam, 1983.

4. Zhang LG, Yu TX. Modified adaptive dynamic relaxation method and its application to elastic-plastic bending and wrinkling of circular plates. Computers and Structures 1989; 33(2):609-614.

5. Haseganu EM, Steigmann DJ. Analysis of partly wrinkled membranes by the method of dynamic relaxation. Computational Mechanics 1994; 14(6):596-614.

6. Hughes TJR, Taylor RL. Unconditionally stable algotiyhms for elastic/ visco-plastic finite element analysis. Computers and Structures 1978; 8(2):169-173.

7. Peric D. On a class of constitutive equations in viscoplasticity: formulation and computational issues. International Journal for Numerical Methods in Engineering 1993; 36(8):1365-1393.

8. Simo JC. Nonlinear stability of the time-discrete variational problem of evolution in nonlinear heat conduction, plasticity and viscoplasticity. Computer Methods in Applied Mechanics and Engineering 1991; 88(1):111-131.

9. Betegon C, del Coz JJ, Penuelas I. Implicit integration procedure for viscoplastic Gurson materials. Computer Methods in Applied Mechanics and Engineering 2006; 195(44-47):6146-6157.

10. Alfano G, Angelis FD, Rosati L. General solution procedures in elasto/viscoplasticity. Computer Methods in Applied Mechanics and Engineering 2001; 190(39):5123-5147.

11. Ponthot JP. Unified stress update algorithms for the numerical simulation of large deformation elasto-plastic and elasto-viscoplastic processes. International Journal of Plasticity 2002; 18(1):91-126. 
12. Wang WM, Sluys LJ, De Borst R. Viscoplasticity for instabilities due to strain softening and strain-rate softening. International Journal for Numerical Methods in Engineering 1997; 40(20):3839-3864.

13. Carosio A, Willam KJ, Etse G. On the consistency of viscoplastic formulations. International Journal of Solids and Structures November 2000; 37(48-50):7349-7369.

14. Heeres OM, Suiker AS, de Borst R. A comparison between the Perzyna viscoplastic model and the Consistency viscoplastic model. European Journal of Mechanics - A/Solids 2002; 21(1):1-12.

15. Lorefice R, Etse G, Carol I. Viscoplastic approach for rate-dependent failure analysis of concrete joints and interfaces. International Journal of Solids and Structures 2008; 45(9):2686-2705.

16. Caballero A, Garolera D, Carol I, Gens A. Viscoplastic multilaminate model for jointed rock with stress-prescribed using a stress-prescribed LEGI scheme. Proceedings of the International Conference on Rock Joints and Jointed Rock Masses, Tucson, AZ, 2009; 1-8.

17. Simo JC, Hughes TJR. Computational Inelasticity, Vol. 7. Springer: Berlin, 1998.

18. Simo JC, Kennedy JG, Govindjee S. Non-smooth multisurface plasticity and viscoplasticity. loading/unloading conditions and numerical algorithms. International Journal for Numerical Methods in Engineering 1988; 26(10):21612185.

19. Regueiro RA, Foster CD. Bifurcation analysis for a ratesensitive, nonassociative, threeinvariant, isotropic/kinematic hardening cap plasticity model for geomaterials: Part I. Small strain. International Journal for Numerical and Analytical Methods in Geomechanics 2011; 35(2):201-225.

20. Willam KJ. Numerical solution of inelastic rate processes. Computers \& Structures 1978; 8(3-4):511-531.

21. Kumar V, Morjaria M, Mukherjee S. Numerical integration of some stiff constitutive models of inelastic deformation. Journal of Engineering Materials and Technology 1980; 102(1):92-96.

22. Chang TY, Chen JY, Chu SC. Viscoplastic finite element analysis by automatic subincrementing technique. Journal of engineering mechanics 1988; 114(1):80-96.

23. Peirce D, Shih CF, Needleman A. A tangent modulus method for rate dependent solids. Computers and Structures 1984; 18(5):875-887.

24. Ju JW. Consistent tangent moduli for a class of viscoplasticity. Journal of Engineering Mechanics 1990; 116(8): 1764-1779.

25. Winkel BV, Gerstle KH, Ko HY. Analysis of time-dependent deformations of openings in salt media. International Journal of Rock Mechanics and Mining Sciences \& Geomechanics Abstracts 1972; 9(2):249-260.

26. Zienkiewicz OC, Pande GN. Time dependent multilaminate model of rocks a numerical study of deformation and failure of rock masses. International Journal for Numerical and Analytical Methods in Geomechanics 1977; 1(3):219247.

27. Desai CS, Samtani NC, Vulliet L. Constitutive modeling and analysis of creeping slopes. Journal of geotechnical engineering 1995; 121(1):43-56.

28. Samtani NC, Desai CS, Vulliet L. An interface model to describe viscoplastic behavior. International Journal for Numerical and Analytical Methods in Geomechanics 1996; 20(4):231-252.

29. Roosta R, Sadaghiani M, Pak A, Saleh Y. Rock joint modeling using a visco-plastic multilaminate model at constant normal load condition. Geotechnical \& Geological Engineering 2006; 24(5):1449-1468.

30. Wittke W. Rock Mechanics: Theory and Applications, with Case Historie. Springer-Verlag: Berlin, 1990.

31. Koiter WT. General Theorems for Elastic-plastic Solids. Sneddon and Hill eds: North-Holland Amsterdam, 1960.

32. Sherman J, Morrison WJ. Adjustment of an inverse matrix corresponding to a change in one element of a given matrix. The Annals of Mathematical Statistics 1950; 21:124-127.

33. Gens A, Carol I, Alonso EE. An interface element formulation for the analysis of soil-reinforcement interaction. Computers and Geotechnics 1989; 7:133-151.

34. Carol I, Prat P, López CM. Normal/shear cracking model: application to discrete crack analysis. Journal of Engineering Mechanics 1997; 123(8):765-773.

35. Caballero A, Willam KJ, Carol I. Consistent tangent formulation for 3D interface modeling of cracking/fracture in quasi-brittle materials. Computer Methods in Applied Mechanics and Engineering 2008; 197(33):2804-2822. 